\title{
Non-commutative Sylvester's determinantal identity
}

\author{
Matjaž Konvalinka \\ Department of Mathematics \\ Massachusetts Institute of Technology, Cambridge, MA 02139, USA \\ konvalinka@math.mit.edu \\ http://www-math.mit.edu/ konvalinka/
}

Submitted: Mar 7, 2007; Accepted: May 19, 2007; Published: May 31, 2007

Mathematics Subject Classifications: 05A30, 15A15

\begin{abstract}
Sylvester's identity is a classical determinantal identity with a straightforward linear algebra proof. We present combinatorial proofs of several non-commutative extensions, and find a $\beta$-extension that is both a generalization of Sylvester's identity and the $\beta$-extension of the quantum MacMahon master theorem.
\end{abstract}

\section{Introduction}

\subsection{Classical Sylvester's determinantal identity.}

Combinatorial linear algebra is a beautiful and underdeveloped part of enumerative combinatorics. The underlying idea is very simple: one takes a matrix identity and views it as an algebraic result over a (possibly non-commutative) ring. Once the identity is translated into the language of words, an explicit bijection or an involution is employed to prove the result. The resulting combinatorial proofs are often insightful and lead to extensions and generalizations of the original identities, often in unexpected directions.

Sylvester's identity is a classical determinantal identity that is usually written in the form used by Bareiss ([B]).

Theorem 1.1 (Sylvester's identity) Let $A$ denote a matrix $\left(a_{i j}\right)_{m \times m}$; take $n<i, j \leq$ $m$ and define

$$
A_{0}=\left(\begin{array}{cccc}
a_{11} & a_{12} & \cdots & a_{1 n} \\
a_{21} & a_{22} & \cdots & a_{2 n} \\
\vdots & \vdots & \ddots & \vdots \\
a_{n 1} & a_{n 2} & \cdots & a_{n n}
\end{array}\right), \quad a_{i *}=\left(\begin{array}{llll}
a_{i 1} & a_{i 2} & \cdots & a_{i n}
\end{array}\right), \quad a_{* j}=\left(\begin{array}{c}
a_{1 j} \\
a_{2 j} \\
\vdots \\
a_{n j}
\end{array}\right),
$$




$$
b_{i j}=\operatorname{det}\left(\begin{array}{cc}
A_{0} & a_{* j} \\
a_{i *} & a_{i j}
\end{array}\right), \quad B=\left(b_{i j}\right)_{n+1 \leq i, j \leq m}
$$

Then

$$
\operatorname{det} A \cdot\left(\operatorname{det} A_{0}\right)^{m-n-1}=\operatorname{det} B \text {. }
$$

EXAMPLE 1.2 If we take $n=1$ and $m=3$, the Sylvester's identity says that

$$
\begin{gathered}
\left(a_{11} a_{22} a_{33}-a_{11} a_{32} a_{23}-a_{21} a_{12} a_{33}+a_{21} a_{32} a_{13}+a_{31} a_{12} a_{23}-a_{31} a_{22} a_{13}\right) a_{11}= \\
=\left|\begin{array}{ll}
a_{11} a_{22}-a_{21} a_{12} & a_{11} a_{23}-a_{21} a_{13} \\
a_{11} a_{32}-a_{31} a_{12} & a_{11} a_{33}-a_{31} a_{13}
\end{array}\right| .
\end{gathered}
$$

Bareiss's proof of Theorem 1.1 is a pretty straightforward linear algebra argument; see $[\mathrm{MG}],[\mathrm{AAM}]$ for other proofs and some mild generalizations.

\subsection{Extensions of Sylvester's identity.}

The Sylvester's identity has been intensely studied, mostly in the algebraic rather than combinatorial context. In 1991, a generalization to quasideterminants, essentially equivalent to our Theorem 3.1, was found by Gelfand and Retakh [GeR]. Krob and Leclerc [KL] used their result to prove the following quantum version. if:

Let $q \in \mathbb{C} \backslash\{0\}$. Call a matrix (in non-commutative variables) $A=\left(a_{i j}\right)_{m \times m}$ quantum

- $a_{j k} a_{i k}=q a_{i k} a_{j k}$ for $i<j$,

- $a_{i l} a_{i k}=q a_{i k} a_{i l}$ for $k<l$,

- $a_{j k} a_{i l}=a_{i l} a_{j k}$ for $i<j, k<l$,

- $a_{i k} a_{j l}-a_{j l} a_{i k}=\left(q^{-1}-q\right) a_{i l} a_{j k}$ for $i<j, k<l$.

Define the quantum determinant of a matrix $A$ by

$$
\operatorname{det}_{q} A=\sum_{\sigma \in S_{m}}(-q)^{-\operatorname{inv} \sigma} a_{\sigma(1) 1} a_{\sigma(2) 2} \cdots a_{\sigma(m) m},
$$

where inv $\sigma$ denotes the number of inversions of the permutation $\sigma$.

Theorem 1.3 (Krob, Leclerc) For a quantum matrix $A=\left(a_{i j}\right)_{m \times m}$, take $n, A_{0}, a_{i *}$ and $a_{* j}$ as before, and define

$$
b_{i j}=\operatorname{det}_{q}\left(\begin{array}{cc}
A_{0} & a_{* j} \\
a_{i *} & a_{i j}
\end{array}\right), \quad B=\left(b_{i j}\right)_{n+1 \leq i, j \leq m} .
$$

Then

$$
\operatorname{det}_{q} A \cdot\left(\operatorname{det}_{q} A_{0}\right)^{m-n-1}=\operatorname{det}_{q} B
$$


Krob and Leclerc's proof consists of an application of the so-called quantum Muir's law of extensible minors to the expansion of a minor.

Since then, Molev found several far-reaching extensions to Yangians, including other root systems [Mo1, Mo2]; see also [HM].

\subsection{Main result.}

In this paper, we find a multiparameter right-quantum analogue of Sylvester's identity. We use the techniques developed in $[\mathrm{KP}]$.

Fix non-zero complex numbers $q_{i j}$ for $1 \leq i<j \leq m$. We call a matrix $A$ q-rightquantum if

$$
\begin{aligned}
a_{j k} a_{i k} & =q_{i j} a_{i k} a_{j k} \text { for all } i<j, \\
a_{i k} a_{j l}-q_{i j}^{-1} a_{j k} a_{i l} & =q_{k l} q_{i j}^{-1} a_{j l} a_{i k}-q_{k l} a_{i l} a_{j k} \text { for all } i<j, k<l .
\end{aligned}
$$

In the next section, we define the concept of a q-determinant of a square matrix. We then have

$$
\operatorname{det}_{\mathbf{q}}(I-A)=\sum_{J \subseteq[m]}(-1)^{|J|} \operatorname{det}_{\mathbf{q}} A_{J}
$$

where

$$
\operatorname{det}_{\mathbf{q}} A_{J}=\sum_{\sigma \in S_{J}}\left(\prod_{p<r: j_{p}>j_{r}} q_{j_{r} j_{p}}^{-1}\right) a_{\sigma\left(j_{1}\right) j_{1}} \cdots a_{\sigma\left(j_{k}\right) j_{k}}
$$

for $J=\left\{j_{1}<j_{2}<\ldots<j_{k}\right\}$.

Our main theorem is the following.

Theorem 1.4 (q-right-quantum Sylvester's determinant identity) Suppose that $A=\left(a_{i j}\right)_{m \times m}$ is a q-right-quantum matrix, and we choose $n<m$. Let $A_{0}, a_{i *}, a_{* j}$ be defined as above, and let

$$
c_{i j}^{\mathbf{q}}=-\operatorname{det}_{\mathbf{q}}^{-1}\left(I-A_{0}\right) \cdot \operatorname{det}_{\mathbf{q}}\left(\begin{array}{cc}
I-A_{0} & -a_{* j} \\
-a_{i *} & -a_{i j}
\end{array}\right), \quad C^{\mathbf{q}}=\left(c_{i j}^{\mathbf{q}}\right)_{n+1 \leq i, j \leq m} .
$$

Suppose $q_{i j}=q_{i^{\prime} j^{\prime}}$ for all $i, i^{\prime} \leq n$ and $j, j^{\prime}>n$. Then

$$
\operatorname{det}_{\mathbf{q}}^{-1}\left(I-A_{0}\right) \cdot \operatorname{det}_{\mathbf{q}}(I-A)=\operatorname{det}_{\mathbf{q}}\left(I-C^{\mathbf{q}}\right) .
$$

The determinant $\operatorname{det}_{\mathbf{q}}\left(I-A_{0}\right)$ does not commute with other determinants in the definition of $c_{i j}^{\mathbf{q}}$, so the identity cannot be written in a form analogous to Theorem 1.1. See Remark 9.9 for a discussion of the necessity of the condition $q_{i j}=q_{i^{\prime} j^{\prime}}$ for $i, i^{\prime} \leq n$, $j, j^{\prime}>n$.

The proof roughly follows the pattern of the proof of the main theorem in [KP]. First we show a combinatorial proof of the classical Sylvester's identity (Sections 3 and 4). Then 
we adapt the proof to simple non-commutative cases - the Cartier-Foata case (Section 5) and the right-quantum case (Section 6). We extend the results to cases with a weight (Sections 7 and 8) and to multiparameter weighted cases (Sections 9 and 10). We also present a $\beta$-extension of Sylvester's identity in Section 11.

\section{Algebraic framework}

\subsection{Words and matrices.}

We work in the $\mathbb{C}$-algebra $\mathcal{A}$ of formal power series in non-commuting variables $a_{i j}, 1 \leq$ $i, j \leq m$. Elements of $\mathcal{A}$ are infinite linear combinations of words in variables $a_{i j}$ (with coefficients in $\mathbb{C}$ ). In most cases we take elements of $\mathcal{A}$ modulo some ideal $\mathcal{I}$ generated by a finite number of quadratic relations. For example, if $\mathcal{I}_{\text {comm }}$ is generated by $a_{i j} a_{k l}=a_{k l} a_{i j}$ for all $i, j, k, l$, then $\mathcal{A} / \mathcal{I}_{\text {comm }}$ is the symmetric algebra (the free commutative algebra with variables $a_{i j}$ ).

We abbreviate the product $a_{\lambda_{1} \mu_{1}} \cdots a_{\lambda_{\ell} \mu_{\ell}}$ to $a_{\lambda, \mu}$ for $\lambda=\lambda_{1} \cdots \lambda_{\ell}$ and $\mu=\mu_{1} \cdots \mu_{\ell}$, where $\lambda$ and $\mu$ are regarded as words in the alphabet $\{1, \ldots, m\}$. For such a word $\nu=\nu_{1} \cdots \nu_{\ell}$, define the set of inversions

$$
\mathcal{I}(\nu)=\left\{(i, j): i<j, \nu_{i}>\nu_{j}\right\}
$$

and let inv $\nu=|\mathcal{I}(\nu)|$ be the number of inversions.

\subsection{Determinants.}

Let $B=\left(b_{i j}\right)_{n \times n}$ be a square matrix with entries in $\mathcal{A}$, i.e. $b_{i j}$ 's are linear combinations of words in $\mathcal{A}$. To define the determinant of $B$, expand the terms of

$$
\sum_{\sigma \in S_{n}}(-1)^{\operatorname{inv}(\sigma)} b_{\sigma_{1} 1} \cdots b_{\sigma_{n} n}
$$

and weight a word $a_{\lambda, \mu}$ with a certain weight $w(\lambda, \mu)$. The resulting expression is called the determinant of $B$ (with respect to $\mathcal{A}$ ). In the usual commutative case, all weights are equal to 1.

In all cases we consider we have $w(\varnothing, \varnothing)=1$. Therefore

$$
\frac{1}{\operatorname{det}(I-A)}=\frac{1}{1-\Sigma}=1+\Sigma+\Sigma^{2}+\ldots
$$

where $\Sigma$ is a certain finite sum of words in $a_{i j}$ and both the left and the right inverse of $\operatorname{det}(I-A)$ are equal to the infinite sum on the right. We can use the fraction notation as above in non-commutative situations. 


\subsection{Paths.}

We consider lattice steps of the form $(x, i) \rightarrow(x+1, j)$ for some $x, i, j \in \mathbb{Z}, 1 \leq i, j \leq m$. We think of $x$ being drawn along the $x$-axis, increasing from left to right, and refer to $i$ and $j$ as the starting height and ending height, respectively. We identify the step $(x, i) \rightarrow(x+1, j)$ with the variable $a_{i j}$. Similarly, we identify a finite sequence of steps with a word in the alphabet $\left\{a_{i j}\right\}, 1 \leq i, j \leq m$, i.e. with an element of the algebra $\mathcal{A}$. If each step in a sequence starts at the ending point of the previous step, we call such a sequence a lattice path. A lattice path with starting height $i$ and ending height $j$ is called a path from $i$ to $j$.

EXAMPLE 2.1 The following is a path from 4 to 4 .

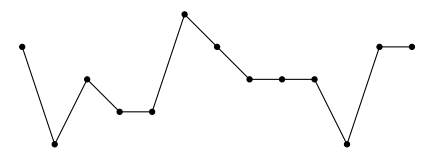

Figure 1: Representation of the word $a_{41} a_{13} a_{32} a_{22} a_{25} a_{54} a_{43} a_{33} a_{33} a_{31} a_{14} a_{44}$.

Recall that the $(i, j)$-th entry of $A^{k}$ is the sum of all paths of length $k$ from $i$ to $j$. Since

$$
(I-A)^{-1}=I+A+A^{2}+\ldots,
$$

the $(i, j)$-th entry of $(I-A)^{-1}$ is the sum of all paths (of any length) from $i$ to $j$.

\section{Non-commutative Sylvester's identity}

As in Section 1, choose $n<m$, and denote the matrix $\left(a_{i j}\right)_{m \times m}$ by $A$ and $\left(a_{i j}\right)_{n \times n}$ by $A_{0}$.

We will show a combinatorial proof of the non-commutative Sylvester's identity due to Gelfand and Retakh, see [GeR].

Theorem 3.1 (Gelfand-Retakh) Consider the matrix $C=\left(c_{i j}\right)_{n+1 \leq i, j \leq m}$, where

$$
c_{i j}=a_{i j}+a_{i *}\left(I-A_{0}\right)^{-1} a_{* j} .
$$

Then

$$
(I-A)_{i j}^{-1}=(I-C)_{i j}^{-1}
$$

Proof: Take a lattice path $a_{i i_{1}} a_{i_{1} i_{2}} \cdots a_{i_{\ell-1} j}$ with $i, j>n$. Clearly it can be uniquely divided into paths $P_{1}, P_{2}, \ldots P_{p}$ with the following properties:

- the ending height of $P_{i}$ is the starting height of $P_{i+1}$

- the starting and the ending heights of all $P_{i}$ are strictly greater than $n$ 
- all intermediate heights are less than or equal to $n$

Next, note that

$$
c_{i j}=a_{i j}+a_{i *}\left(I-A_{0}\right)^{-1} a_{* j}=a_{i j}+\sum_{k, l \leq n} a_{i k}\left(I+A_{0}+A_{0}^{2}+\ldots\right)_{k l} a_{l j}
$$

is the sum over all non-trivial paths with starting height $i$, ending height $j$, and intermediate heights $\leq n$. This decomposition hence proves the theorem.

ExAmple 3.2 The following figure depicts the path from Example 2.1 with a dotted line between heights $n$ and $n+1$, and the corresponding decomposition, for $n=3$.

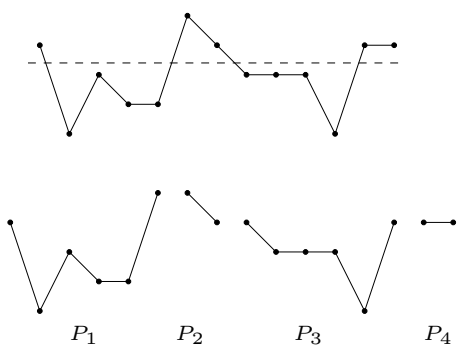

Figure 2: The decomposition $\left(a_{41} a_{13} a_{32} a_{22} a_{25}\right)\left(a_{54}\right)\left(a_{43} a_{33} a_{33} a_{31} a_{14}\right)\left(a_{44}\right)$.

The theorem implies that

$$
\begin{gathered}
(I-A)_{n+1, n+1}^{-1}\left(I-A^{n+1, n+1}\right)_{n+2, n+2}^{-1} \cdots\left(I-\left(\begin{array}{cc}
A_{0} & a_{* m} \\
a_{m *} & a_{m m}
\end{array}\right)\right)_{m m}^{-1}= \\
=(I-C)_{n+1, n+1}^{-1}\left(I-C^{n+1, n+1}\right)_{n+2, n+2}^{-1} \cdots\left(1-c_{m m}\right)^{-1} .
\end{gathered}
$$

Here $A^{n+1, n+1}$ is the matrix $A$ with the $(n+1)$-th row and column removed.

In all the cases we consider in the following sections, both the left-hand side and the right-hand side of this equation can be written in terms of determinants, as in the classical Sylvester's identity.

\section{Commutative case}

Recall that if $D$ is an invertible matrix with commuting entries, we have

$$
\left(D^{-1}\right)_{i j}=(-1)^{i+j} \frac{\operatorname{det} D^{j i}}{\operatorname{det} D}
$$

where $D^{j i}$ denotes the matrix $D$ without the $j$-th row and the $i$-th column. Apply this to (3.1): the numerators (except the last one on the left-hand side) and denominators (except the first one on both sides) cancel each other, and we get

$$
\frac{\operatorname{det}\left(I-A_{0}\right)}{\operatorname{det}(I-A)}=\frac{1}{\operatorname{det}(I-C)} \text {. }
$$


Proposition 4.1 For $i, j>n$ we have

$$
\delta_{i j}-c_{i j}=\frac{\operatorname{det}\left(\begin{array}{cc}
I-A_{0} & -a_{* j} \\
-a_{i *} & \delta_{i j}-a_{i j}
\end{array}\right)}{\operatorname{det}\left(I-A_{0}\right)} .
$$

Proof: Clearly we have

$$
\left(1-c_{i j}\right)^{-1}=\left(\left(I-\left(\begin{array}{cc}
A_{0} & a_{* j} \\
a_{i *} & a_{i j}
\end{array}\right)\right)^{-1}\right)_{i j}
$$

and by (4.1), this is equal to

$$
\frac{\operatorname{det}\left(I-A_{0}\right)}{\operatorname{det}\left(I-\left(\begin{array}{cc}
A_{0} & a_{* j} \\
a_{i *} & a_{i j}
\end{array}\right)\right)}
$$

This finishes the proof for $i=j$, and for $i \neq j$ we have

$$
\begin{aligned}
1-c_{i j} & =\frac{\operatorname{det}\left(\begin{array}{cc}
I-A_{0} & -a_{* j} \\
-a_{i *} & 1-a_{i j}
\end{array}\right)}{\operatorname{det}\left(I-A_{0}\right)}=\frac{\operatorname{det}\left(\begin{array}{cc}
I-A_{0} & -a_{* j} \\
-a_{i *} & -a_{i j}
\end{array}\right)+\operatorname{det}\left(\begin{array}{cc}
I-A_{0} & 0 \\
-a_{i *} & 1
\end{array}\right)}{\operatorname{det}\left(I-A_{0}\right)}= \\
= & \frac{\operatorname{det}\left(\begin{array}{cc}
I-A_{0} & -a_{* j} \\
-a_{i *} & -a_{i j}
\end{array}\right)+\operatorname{det}\left(I-A_{0}\right)}{\operatorname{det}\left(I-A_{0}\right)}=\frac{\operatorname{det}\left(\begin{array}{cc}
I-A_{0} & -a_{* j} \\
-a_{i *} & -a_{i j}
\end{array}\right)}{\operatorname{det}\left(I-A_{0}\right)}+1 .
\end{aligned}
$$

Proof (of Theorem 1.1): The proposition, together with (4.1), implies that

$$
\frac{\operatorname{det}(I-A)}{\operatorname{det}\left(I-A_{0}\right)}=\operatorname{det}(I-C)=\operatorname{det}\left(I-A_{0}\right)^{n-m} \operatorname{det} B
$$

for

$$
b_{i j}=\operatorname{det}\left(\begin{array}{cc}
I-A_{0} & -a_{* j} \\
-a_{i *} & \delta_{i j}-a_{i j}
\end{array}\right), \quad B=\left(b_{i j}\right)_{n+1 \leq i, j \leq m},
$$

which is Theorem 1.1 for the matrix $I-A$.

\section{Cartier-Foata case}

A matrix $A$ is Cartier-Foata if

$$
a_{i k} a_{j l}=a_{j l} a_{i k}
$$

for $i \neq j$, and right-quantum if

$$
\begin{aligned}
a_{j k} a_{i k} & =a_{i k} a_{j k} \text { for all } i \neq j, \\
a_{i k} a_{j l}-a_{j k} a_{i l} & =a_{j l} a_{i k}-a_{i l} a_{j k} \text { for all } i \neq j, k \neq l .
\end{aligned}
$$


Cartier-Foata matrices were introduced in $[\mathrm{CF}]$ and further studied in $[\mathrm{F} 2]$; see also [GGRW, §3.9]. For references on quantum and right-quantum algebras, see [K] and [M3].

A Cartier-Foata matrix is also right-quantum, but the proofs tend to be much simpler for Cartier-Foata matrices.

Note also that the classical definition of the determinant

$$
\operatorname{det} B=\sum_{\sigma \in S_{m}}(-1)^{\operatorname{inv} \sigma} b_{\sigma_{1} 1} \cdots b_{\sigma_{m} m}
$$

makes sense for a matrix $B=\left(b_{i j}\right)_{m \times m}$ with entries generated by $a_{i j}$; in the language of Section 2 , we have $w(\lambda, \mu)=1$ for all words $\lambda, \mu$.

A special case (when $i=j=1$ ) of the following proposition is [KP, Proposition 3.2, Proposition 4.2]. The proof in this more general case is almost exactly the same and we omit it.

Proposition 5.1 If $A=\left(a_{i j}\right)_{m \times m}$ is a Cartier-Foata matrix or a right-quantum matrix, we have

$$
\left(\frac{1}{I-A}\right)_{i j}=(-1)^{i+j} \frac{1}{\operatorname{det}(I-A)} \cdot \operatorname{det}(I-A)^{j i}
$$

for all $i, j$.

Lemma 5.2 If $A$ is a Cartier-Foata matrix, $C$ is a right-quantum matrix.

Proof: Choose $i, j, k>n, i \neq j$. The product $c_{i k} c_{j k}$ is the sum of terms of the form

$$
a_{i i_{1}} a_{i_{1} i_{2}} \cdots a_{i_{p} k} a_{j j_{1}} a_{j_{1} j_{2}} \cdots a_{j_{r} k}
$$

for $p, r \geq 0, i_{1}, \ldots, i_{p}, j_{1}, \ldots, j_{r} \leq n$. Note that with the (possible) exception of $i, j, k$, all other terms appear as starting heights exactly as many times as they appear as ending heights.

Identify this term with a sequence of steps, as described in Section 2. We will perform a series of switches of steps that will transform such a term into a term of $c_{j k} c_{i k}$.

The variable $a_{j j_{1}}$ (or $a_{j k}$ if $r=0$ ) commutes with all variables that appear before it. In other words, in the algebra $\mathcal{A}$, the expressions

$$
a_{i i_{1}} a_{i_{1} i_{2}} \cdots a_{i_{p} k} a_{j j_{1}} a_{j_{1} j_{2}} \cdots a_{j_{r} k}
$$

and

$$
a_{j j_{1}} a_{i i_{1}} a_{i_{1} i_{2}} \cdots a_{i_{p} k} a_{j_{1} j_{2}} \cdots a_{j_{r} k}
$$

are the same modulo the ideal $\mathcal{I}_{\text {cf }}$ generated by $a_{i k} a_{j l}-a_{j l} a_{i k}$ for $i \neq j$. Graphically, we can keep switching the step $j \rightarrow j_{1}$ with the step to its left until it is at the beginning of the sequence.

If $r=0$, we are already done. If not, take the first step to the right of $a_{j j_{1}}$ that has starting height $j_{1}$; such a step certainly exists - for example $j_{1} \rightarrow j_{2}$. Without changing 
the expression modulo $\mathcal{I}_{\text {cf }}$, we can switch this step with the ones to the left until it is just right of $j \rightarrow j_{1}$. Continue this procedure; eventually, our sequence is transformed into an expression of the form

$$
a_{j j_{1}^{\prime}} a_{j_{1}^{\prime} j_{2}^{\prime}} \cdots a_{j_{r^{\prime}}^{\prime}} a_{i i_{1}^{\prime}} a_{i_{1}^{\prime} i_{2}^{\prime}} \cdots a_{i_{p^{\prime}} k}
$$

which is equal modulo $\mathcal{I}_{\text {cf }}$ to the expression we started with.

As an example, take $m=5, n=2, i=3, j=5, k=4$ and the term $a_{31} a_{12} a_{24} a_{52} a_{22} a_{24}$. The steps shown in Figure 3 transform it into $a_{52} a_{24} a_{31} a_{12} a_{22} a_{24}$.

It is clear that applying the same procedure to the result, but with the roles of $i$ 's and $j$ 's interchanged, gives the original sequence. This proves that indeed $c_{i k} c_{j k}=c_{j k} c_{i k}$.

The proof of the other relation (5.3) is similar and we only sketch it. Choose $i, j, k, l>n$, $i \neq j, k \neq l$. Then $c_{i k} c_{j l}+c_{i l} c_{j k}$ is the sum of terms of the form

$$
a_{i i_{1}} a_{i_{1} i_{2}} \cdots a_{i_{p} k} a_{j j_{1}} a_{j_{1} j_{2}} \cdots a_{j_{r} l}
$$

and of the form

$$
a_{i i_{1}} a_{i_{1} i_{2}} \cdots a_{i_{p} l} a_{j j_{1}} a_{j_{1} j_{2}} \cdots a_{j_{r} k}
$$

for $p, r \geq 0, i_{1}, \ldots, i_{p}, j_{1}, \ldots, j_{r} \leq n$. Applying the same procedure as above to the first term yields either

$$
a_{j j_{1}^{\prime}} a_{j_{1}^{\prime} j_{2}^{\prime}} \cdots a_{j_{r^{\prime}}^{\prime} k} a_{i i_{1}^{\prime}} a_{i_{1}^{\prime} i_{2}^{\prime}} \cdots a_{i_{p^{\prime}}^{\prime} l}
$$

or

$$
a_{j j_{1}^{\prime}} a_{j_{1}^{\prime} j_{2}^{\prime}} \cdots a_{j_{r^{\prime}}^{\prime}} a_{i i_{1}^{\prime}} a_{i_{1}^{\prime} i_{2}^{\prime}} \cdots a_{i_{p^{\prime}}^{\prime} k}
$$

this procedure is reversible and it yields the desired identity. See Figure 4 for examples with $m=5, n=2, i=3, j=4, k=3, l=5$.

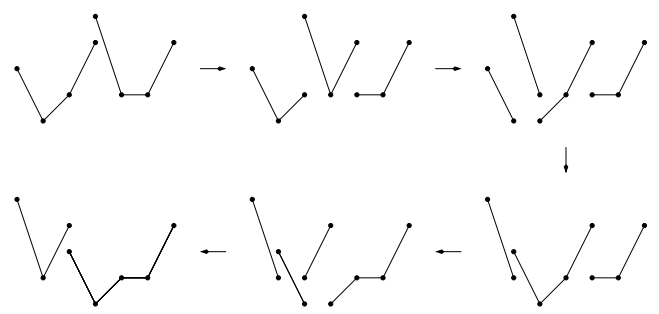

Figure 3: Transforming $a_{31} a_{12} a_{24} a_{52} a_{22} a_{24}$ into $a_{52} a_{24} a_{31} a_{12} a_{22} a_{24}$.

If $A$ is Cartier-Foata, Proposition 5.1 implies

$$
(I-A)_{n+1, n+1}^{-1}\left(I-A^{n+1, n+1}\right)_{n+2, n+2}^{-1} \cdots=\operatorname{det}^{-1}(I-A) \cdot \operatorname{det}\left(I-A_{0}\right) .
$$

By Lemma 5.2, $C$ is right-quantum, so by Proposition 5.1

$$
(I-C)_{n+1, n+1}^{-1}\left(I-C^{n+1, n+1}\right)_{n+2, n+2}^{-1} \cdots=\operatorname{det}^{-1}(I-C),
$$




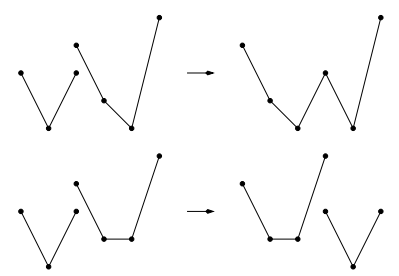

Figure 4: Transforming $a_{31} a_{13} a_{42} a_{21} a_{15}$ and $a_{31} a_{13} a_{42} a_{22} a_{25}$.

and hence

$$
\operatorname{det}^{-1}\left(I-A_{0}\right) \cdot \operatorname{det}(I-A)=\operatorname{det}(I-C) .
$$

In the classical Sylvester's identity, the entries of $I-C$ are also expressed as determinants. The following is an analogue of Proposition 4.1.

Proposition 5.3 If $A$ is Cartier-Foata, then

$$
c_{i j}=-\operatorname{det}^{-1}\left(I-A_{0}\right) \cdot \operatorname{det}\left(\begin{array}{cc}
I-A_{0} & -a_{* j} \\
-a_{i *} & -a_{i j}
\end{array}\right) .
$$

Proof: We can repeat the proof of Proposition 4.1 almost verbatim. We have

$$
\left(1-c_{i j}\right)^{-1}=\left(\left(I-\left(\begin{array}{cc}
A_{0} & a_{* j} \\
a_{i *} & a_{i j}
\end{array}\right)\right)^{-1}\right)_{i j},
$$

and because the matrix

$$
\left(\begin{array}{cc}
A_{0} & a_{* j} \\
a_{i *} & a_{i j}
\end{array}\right)
$$

is still Cartier-Foata, Proposition 5.1 shows that this is equal to

$$
\operatorname{det}^{-1}\left(I-\left(\begin{array}{cc}
A_{0} & a_{* j} \\
a_{i *} & a_{i j}
\end{array}\right)\right) \cdot \operatorname{det}\left(I-A_{0}\right)
$$

We get

$$
\begin{gathered}
1-c_{i j}=\operatorname{det}^{-1}\left(I-A_{0}\right) \cdot \operatorname{det}\left(I-\left(\begin{array}{cc}
A_{0} & a_{* j} \\
a_{i *} & a_{i j}
\end{array}\right)\right)= \\
=\operatorname{det}^{-1}\left(I-A_{0}\right) \cdot\left(\operatorname{det}\left(\begin{array}{cc}
I-A_{0} & -a_{* j} \\
-a_{i *} & -a_{i j}
\end{array}\right)+\operatorname{det}\left(\begin{array}{cc}
I-A_{0} & 0 \\
-a_{i *} & 1
\end{array}\right)\right)= \\
=\operatorname{det}^{-1}\left(I-A_{0}\right) \cdot\left(\operatorname{det}\left(\begin{array}{cc}
I-A_{0} & -a_{* j} \\
-a_{i *} & -a_{i j}
\end{array}\right)+\operatorname{det}\left(I-A_{0}\right)\right)= \\
=\operatorname{det}^{-1}\left(I-A_{0}\right) \cdot \operatorname{det}\left(\begin{array}{cc}
I-A_{0} & -a_{* j} \\
-a_{i *} & -a_{i j}
\end{array}\right)+1 .
\end{gathered}
$$

We have proved the following. 
Theorem 5.4 (Cartier-Foata Sylvester's identity) Let $A=\left(a_{i j}\right)_{m \times m}$ be a CartierFoata matrix, and choose $n<m$. Let $A_{0}, a_{i *}, a_{* j}$ be defined as above, and let

$$
c_{i j}=-\operatorname{det}^{-1}\left(I-A_{0}\right) \cdot \operatorname{det}\left(\begin{array}{cc}
I-A_{0} & -a_{* j} \\
-a_{i *} & -a_{i j}
\end{array}\right), \quad C=\left(c_{i j}\right)_{n+1 \leq i, j \leq m} .
$$

Then

$$
\operatorname{det}^{-1}\left(I-A_{0}\right) \cdot \operatorname{det}(I-A)=\operatorname{det}(I-C) .
$$

\section{Right-quantum analogue}

The right-quantum version of the Sylvester's identity is very similar; we prove a rightquantum version of Lemma 5.2 and Proposition 5.3, and a right-quantum version of Theorem 5.4 follows.

The only challenging part is the following.

Lemma 6.1 If $A$ is a right-quantum matrix, so is $C$.

Proof: Choose $i, j, k>n, i \neq j$. Instead of dealing directly with the equality $c_{i k} c_{j k}=$ $c_{j k} c_{i k}$, we will prove an equivalent identity.

Denote by $\mathcal{P}_{i j}^{k}\left(k_{1}, k_{2}, \ldots, k_{n}\right)$ the set of sequences of $k_{1}+\ldots+k_{n}+2$ steps with the following properties:

- starting heights form a non-decreasing sequence;

- each $r$ between 1 and $n$ appears exactly $k_{r}$ times as a starting height and exactly $k_{r}$ times as an ending height;

- $i$ and $j$ appear exactly once as starting heights;

- $k$ appears exactly twice as an ending height.

For $m=5, n=2, i=3, j=5, k=4, k_{1}=1, k_{2}=1$, all such sequences are shown in Figure 5 .

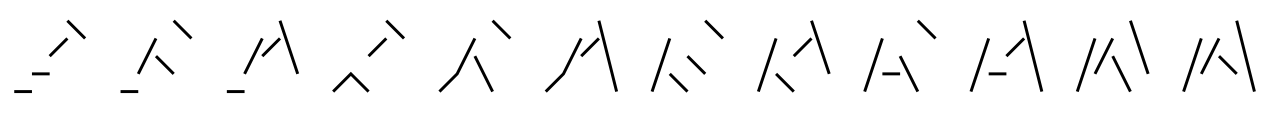

Figure 5: Sequences in the set $\mathcal{P}_{35}^{4}(1,1)$.

We will do something very similar to the proof of Lemma 5.2: we will perform switches on sequences in $\mathcal{P}_{i j}^{k}\left(k_{1}, k_{2}, \ldots, k_{n}\right)$ until they are transformed into sequences of the form $P_{1} P_{2} P_{3}$, where:

- $P_{1}$ is a path from $i$ to $k$ with all intermediate heights $\leq n$; 
- $P_{2}$ is a path from $j$ to $k$ with all intermediate heights $\leq n$;

- $P_{3}$ is a sequence of steps with non-decreasing heights, with all heights $\leq n$, and with the number of steps with starting height $r$ equal to the number of steps with ending height $r$ for all $r$.

Namely, we move the step $i \rightarrow i^{\prime}$ to the first place, the first step of the form $i^{\prime} \rightarrow i^{\prime \prime}$ to the second place, etc. If we start with $\alpha \in \mathcal{P}_{i j}^{k}\left(k_{1}, k_{2}, \ldots, k_{n}\right)$, we denote the sequences we get during this process by $\alpha, \psi(\alpha), \psi^{2}(\alpha), \ldots, \psi^{N}(\alpha)$, the final result $\psi^{N}(\alpha)$ is denoted by $\varphi(\alpha)$, and we take $\psi^{N+l}(\alpha)=\psi^{N}(\alpha)$ for all $l \geq 0$. For example, the sequence $a_{11} a_{24} a_{34} a_{52}$ is transformed into $a_{34} a_{52} a_{24} a_{11}$ in 5 steps, see Figure 6.

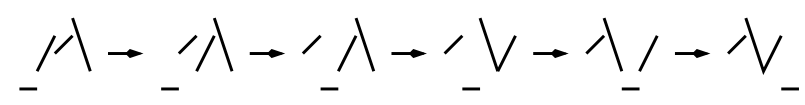

Figure 6: Transforming $a_{11} a_{24} a_{34} a_{52}$ into $a_{34} a_{52} a_{24} a_{11}$.

Of course, we have to prove that this can be done without changing the sum modulo the ideal $\mathcal{I}_{\text {rq }}$ generated by relations (5.2)-(5.3), and this is done in almost exactly the same way as the proof in [KP, §4]. Figure 7 is an example for $m=5, n=2, i=3, j=5, k=$ $4, k_{1}=1, k_{2}=1$; each column corresponds to a transformation of an element of $\mathcal{P}_{35}^{4}(1,1)$, if two elements in the same row have the same label, their sum can be transformed into the sum of the corresponding elements in the next row by use of the relation (5.3), and if an element is not labeled it either means that it is transformed into the corresponding element in the next row by use of the relation (5.2) or is already in the required form.

To prove this can be done in general, define the rank of a sequence $a_{i_{1} j_{1}} a_{i_{2} j_{2}} \cdots$ to be the cardinality of $\left\{(k, l): k<l, i_{k}>i_{l}\right\}$. Clearly, the rank of an element of $\mathcal{P}=$ $\mathcal{P}_{i j}^{k}\left(k_{1}, k_{2}, \ldots, k_{n}\right)$ is 0 , and $\operatorname{rank} \psi^{i+1}(\alpha)=\operatorname{rank} \psi^{i}(\alpha)+1$.

Take $r \geq 0$, and assume that

$$
\sum_{\alpha \in \mathcal{P}} \psi^{r}(\alpha)=\sum_{\alpha \in \mathcal{P}} \alpha
$$

modulo $\mathcal{I}_{\text {rq }}$. Assume that we switch the steps $\left(x-1, i^{\prime}\right) \rightarrow\left(x, k^{\prime}\right)$ and $\left(x, j^{\prime}\right) \rightarrow\left(x+1, l^{\prime}\right)$ in order to get $\psi^{r+1}(\alpha)$ from $\psi^{r}(\alpha)$. If $k^{\prime}=l^{\prime}, \psi^{r+1}(\alpha)=\psi^{r}(\alpha) \bmod \mathcal{I}_{\text {rq }}$ by (5.2). On the other hand, if $k^{\prime} \neq l^{\prime}$, replace $\left(x-1, i^{\prime}\right) \rightarrow\left(x, k^{\prime}\right)$ and $\left(x, j^{\prime}\right) \rightarrow\left(x+1, l^{\prime}\right)$ in $\psi^{r}(\alpha)$ by $\left(x-1, i^{\prime}\right) \rightarrow\left(x, l^{\prime}\right)$ and $\left(x, j^{\prime}\right) \rightarrow\left(x+1, k^{\prime}\right)$; this sequence has rank $r$ and is equal to $\psi^{r}(\beta)$ for some $\beta \in \mathcal{P}$. But then (5.3) tells us that, modulo $\mathcal{I}_{\text {rq }}, \psi^{r+1}(\alpha)+\psi^{r+1}(\beta)=$ $\psi^{r}(\alpha)+\psi^{r}(\beta)$, and so

$$
\sum_{\alpha \in \mathcal{P}} \psi^{r+1}(\alpha)=\sum_{\alpha \in \mathcal{P}} \alpha
$$

modulo $\mathcal{I}_{\text {rq }}$, and by induction

$$
\sum_{\alpha \in \mathcal{P}} \alpha=c_{i k} c_{j k} S
$$

modulo $\mathcal{I}_{\mathrm{rq}}$, where $S$ is the sum over all sequences of steps with the following properties: 


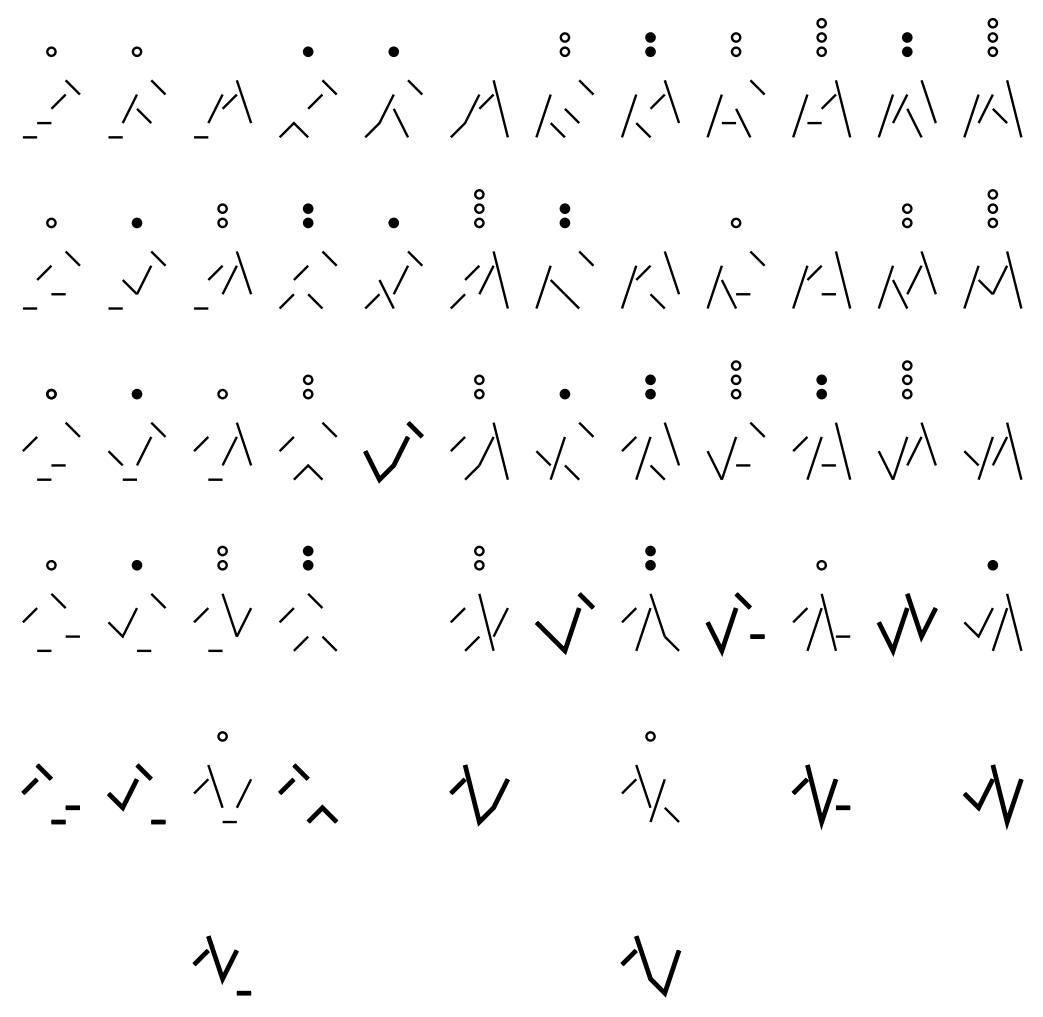

Figure 7: Transforming the sequences in $\mathcal{P}_{35}^{4}(1,1)$ into terms of $c_{34} c_{54} S$.

- starting heights form a non-decreasing sequence;

- starting and ending heights are all between 1 and $n$;

- each $r$ between 1 and $n$ appears as many times as a starting height as an ending height.

Of course, we can also reverse the roles of $i$ and $j$, and this proves that the sum of all elements of $\mathcal{P}_{i j}^{k}\left(k_{1}, k_{2}, \ldots, k_{n}\right)$ is modulo $\mathcal{I}_{\text {rq }}$ also equal to

$$
c_{j k} c_{i k} S \text {. }
$$

Hence, modulo $\mathcal{I}_{\text {rq }}$,

$$
c_{i k} c_{j k} S=c_{j k} c_{i k} S .
$$

But $S=1+a_{11}+\ldots+a_{n n}+a_{11} a_{22}+a_{12} a_{21}+\ldots$ is an invertible element of $\mathcal{A}$, so (6.1) implies

$$
c_{i k} c_{j k}=c_{j k} c_{i k},
$$

provided $A$ is a right-quantum matrix.

The proof of the other relation is almost completely analogous. Now we take $i \neq j, k \neq l$, and define $\mathcal{P}_{i j}^{k l}\left(k_{1}, k_{2}, \ldots, k_{n}\right)$ as the set of sequences of $k_{1}+\ldots+k_{n}+2$ steps with the following properties: 
- starting heights form a non-decreasing sequence;

- each $r$ between 1 and $n$ appears exactly $k_{r}$ times as a starting height and exactly $k_{r}$ times as an ending height;

- $i$ and $j$ appear exactly once as starting heights;

- $k$ and $l$ appear exactly once as ending heights.

A similar reasoning shows that the sum over all elements of $\mathcal{P}_{i j}^{k l}\left(k_{1}, k_{2}, \ldots, k_{n}\right)$ is equal both to $\left(c_{i k} c_{j l}+c_{i l} c_{j k}\right) S$ and to $\left(c_{j l} c_{i k}+c_{j k} c_{i l}\right) S$ modulo $\mathcal{I}_{\text {rq }}$, which implies $c_{i k} c_{j l}+c_{i l} c_{j k}=$ $c_{j l} c_{i k}+c_{j k} c_{i l}$.

Proposition 6.2 If $A$ is right-quantum, then

$$
c_{i j}=-\operatorname{det}^{-1}\left(I-A_{0}\right) \cdot \operatorname{det}\left(\begin{array}{cc}
I-A_{0} & -a_{* j} \\
-a_{i *} & -a_{i j}
\end{array}\right) .
$$

Proof: The proof is exactly the same as the proof of Proposition 5.3.

Theorem 6.3 (right-quantum Sylvester's identity) Let $A=\left(a_{i j}\right)_{m \times m}$ be a rightquantum matrix, and choose $n<m$. Let $A_{0}, a_{i *}, a_{* j}$ be defined as above, and let

$$
c_{i j}=-\operatorname{det}^{-1}\left(I-A_{0}\right) \cdot \operatorname{det}\left(\begin{array}{cc}
I-A_{0} & -a_{* j} \\
-a_{i *} & -a_{i j}
\end{array}\right), \quad C=\left(c_{i j}\right)_{n+1 \leq i, j \leq m} .
$$

Then

$$
\operatorname{det}^{-1}\left(I-A_{0}\right) \cdot \operatorname{det}(I-A)=\operatorname{det}(I-C)
$$

\section{7 q-Cartier-Foata analogue}

Let us find a quantum extension of Theorem 5.4. Fix $q \in \mathbb{C} \backslash\{0\}$. We say that a matrix $A=\left(a_{i j}\right)_{m \times m}$ is $q$-Cartier-Foata if

$$
\begin{aligned}
a_{j l} a_{i k} & =a_{i k} a_{j l} \text { for } i<j, k<l, \\
a_{j l} a_{i k} & =q^{2} a_{i k} a_{j l} \text { for } i<j, k>l, \\
a_{j k} a_{i k} & =q a_{i k} a_{j k} \text { for } i<j,
\end{aligned}
$$

and $q$-right-quantum if

$$
\begin{aligned}
a_{j k} a_{i k} & =q a_{i k} a_{j k} \text { for all } i<j \\
a_{i k} a_{j l}-q^{-1} a_{j k} a_{i l} & =a_{j l} a_{i k}-q a_{i l} a_{j k} \text { for all } i<j, k<l .
\end{aligned}
$$

Clearly, Cartier-Foata and right-quantum matrices are special cases of $q$-Cartier-Foata and $q$-right-quantum matrices, for $q=1$; furthermore, a quantum matrix is also rightquantum. In [GLZ], the term "right quantum" stands for what we call "q-right-quantum". For references, see $[\mathrm{K}]$ and [M3]. 
In the following two sections, the weight $w(\lambda, \mu)$ is equal to $q^{\text {inv } \mu-\operatorname{inv} \lambda}$. For example,

$$
\operatorname{det}_{q}(I-A)=\sum_{J \subseteq[m]}(-1)^{|J|} \operatorname{det}_{q} A_{J}
$$

where

$$
\operatorname{det}_{q} A_{J}=\operatorname{det}_{q}\left(a_{i j}\right)_{i, j \in J}=\sum_{\sigma \in S_{J}}(-q)^{-\operatorname{inv} \sigma} a_{\sigma\left(j_{1}\right) j_{1}} \cdots a_{\sigma\left(j_{k}\right) j_{k}}
$$

for $J=\left\{j_{1}<j_{2}<\ldots<j_{k}\right\}$.

The following extends Proposition 5.1. A special case (when $i=j=1$ ) is $[\mathrm{KP}$, Proposition 5.2, Proposition 6.2]. The proof in this more general case is almost exactly the same and we omit it.

Proposition 7.1 If $A=\left(a_{i j}\right)_{m \times m}$ is a q-Cartier-Foata or a q-right-quantum matrix, we have

$$
\left(\frac{1}{I-A_{[i j]}}\right)_{i j}=(-1)^{i+j} \frac{1}{\operatorname{det}_{q}(I-A)} \cdot \operatorname{det}_{q}(I-A)^{j i}
$$

for all $i, j$, where

$$
A_{[i j]}=\left(\begin{array}{cccccc}
q^{-1} a_{11} & \cdots & q^{-1} a_{1 j} & a_{1, j+1} & \cdots & a_{1 m} \\
\vdots & \ddots & \vdots & \vdots & \ddots & \vdots \\
q^{-1} a_{i-1,1} & \cdots & q^{-1} a_{i-1, j} & a_{i-1, j+1} & \cdots & a_{i-1, m} \\
a_{i 1} & \cdots & a_{i j} & q a_{i, j+1} & \cdots & q a_{i, m} \\
\vdots & \ddots & \vdots & \vdots & \ddots & \vdots \\
a_{m 1} & \cdots & a_{m j} & q a_{m, j+1} & \cdots & q a_{m m}
\end{array}\right) .
$$

We use Theorem 3.1 for $A_{[i j]}$. Let us find the corresponding $C=\left(c_{i^{\prime} j^{\prime}}^{\prime}\right)_{n+1 \leq i^{\prime}, j^{\prime} \leq m}$. Denote

$$
a_{i^{\prime} j^{\prime}}+q^{-1} a_{i^{\prime} *}\left(I-q^{-1} A_{0}\right)^{-1} a_{* j^{\prime}}
$$

by $c_{i^{\prime} j^{\prime}}$ for $i^{\prime}, j^{\prime}>n$. If $i^{\prime}<i, j^{\prime} \leq j$, we have

$$
c_{i^{\prime} j^{\prime}}^{\prime}=q^{-1} a_{i^{\prime} j^{\prime}}+\left(q^{-1} a_{i^{\prime} *}\right)\left(I-q^{-1} A_{0}\right)^{-1}\left(q^{-1} a_{* j^{\prime}}\right)=q^{-1} c_{i^{\prime} j^{\prime}} ;
$$

if $i^{\prime}<i, j^{\prime}>j$, we have

$$
c_{i^{\prime} j^{\prime}}^{\prime}=a_{i^{\prime} j^{\prime}}+\left(q^{-1} a_{i^{\prime} *}\right)\left(I-q^{-1} A_{0}\right)^{-1} a_{* j^{\prime}}=c_{i^{\prime} j^{\prime}}
$$

if $i^{\prime} \geq i, j^{\prime} \leq j$, we have

$$
c_{i^{\prime} j^{\prime}}^{\prime}=a_{i^{\prime} j^{\prime}}+a_{i^{\prime} *}\left(I-q^{-1} A_{0}\right)^{-1}\left(q^{-1} a_{* j^{\prime}}\right)=c_{i^{\prime} j^{\prime}}
$$

and if $i^{\prime} \geq i, j^{\prime}>j$, we have

$$
c_{i^{\prime} j^{\prime}}^{\prime}=q a_{i^{\prime} j^{\prime}}+a_{i^{\prime} *}\left(I-q^{-1} A_{0}\right)^{-1} a_{* j^{\prime}}=q c_{i^{\prime} j^{\prime}} .
$$

We have proved the following. 
Proposition 7.2 With $A_{[i j]}$ as above and with $C=\left(c_{i^{\prime} j^{\prime}}\right)_{n+1 \leq i^{\prime}, j^{\prime} \leq m}$ for

$$
c_{i^{\prime} j^{\prime}}=a_{i^{\prime} j^{\prime}}+a_{i^{\prime} *}\left(I-q^{-1} A_{0}\right)^{-1}\left(q^{-1} a_{* j^{\prime}}\right),
$$

we have

$$
\left(I-A_{[i j]}\right)_{i^{\prime} j^{\prime}}^{-1}=\left(I-C_{[i j]}\right)_{i^{\prime} j^{\prime}}^{-1}
$$

REMARK 7.3 Let us present a slightly different proof of the proposition. Another way to characterize $A_{[i j]}$ is to say that the entry $a_{k l}$ has weight $q$ to the power of

$$
\left\{\begin{array}{l}
1: l>j \\
0: l \leq j
\end{array}-\left\{\begin{array}{l}
1: k<i \\
0: k \geq i
\end{array} .\right.\right.
$$

That means that in $\left(A_{[i j]}^{\ell}\right)_{i_{1} i_{\ell}}$,

$$
a_{i_{1} i_{2}} a_{i_{2} i_{3}} \cdots a_{i_{\ell-1} i_{\ell}}
$$

has weight

$$
q^{\left|\left\{r: i_{r}>j\right\}\right|-\left|\left\{r: i_{r}<i\right\}\right|} .
$$

Assume that we have a decomposition of a path of length $\ell$ from $i^{\prime}$ to $j^{\prime}, i^{\prime}, j^{\prime}>n$, as in Section 3, say $a_{\lambda, \mu}=a_{i^{\prime} \lambda_{1}, \lambda_{1} i_{1}} a_{i_{1} \lambda_{2}, \lambda_{2} i_{2}} \cdots a_{i_{p-1} \lambda_{p}, \lambda_{p} j^{\prime}}$, with all elements of $\lambda_{r}$ at most $n$, $i_{r}>n$, and the length of $\lambda_{r}$ equal to $\ell_{r}$. Put $i_{0}=i^{\prime}, i_{p+1}=j^{\prime}$. The number of indices of $\lambda=i^{\prime} \lambda_{1} \ldots \lambda_{p}$ that are strictly smaller than $i$ is clearly

$$
\sum_{r=1}^{p} \ell_{r}+\left|\left\{r: i_{r}<i\right\}\right|=\ell-p+\left|\left\{r: i_{r}<i\right\}\right|,
$$

and the number of indices of $\mu=\lambda_{1} \ldots \lambda_{p} j^{\prime}$ that are strictly greater than $j$ is $\left|\left\{r: i_{r}>j\right\}\right|$. Therefore the path $a_{\lambda, \mu}$ is weighted by

$$
q^{-\ell+p+\left|\left\{r: i_{r}>j\right\}\right|-\left|\left\{r: i_{r}<i\right\}\right|} .
$$

On the other hand, take a term $a_{\lambda, \mu}=a_{i^{\prime} \lambda_{1}, \lambda_{1} i_{1}} a_{i_{1} \lambda_{2}, \lambda_{2} i_{2}} \cdots a_{i_{p-1} \lambda_{p}, \lambda_{p} j^{\prime}}$ (with $\lambda_{r}, i_{r}$, $\ell_{r}$ as before) of $\left(C_{[i j]}^{\ell}\right)_{i^{\prime} j^{\prime}}$. Each $a_{i_{r-1} \lambda_{r}, \lambda_{r} i_{r}}$ has weight $q^{-\ell_{r}}$ as an element of $C$, and $a_{\lambda, \mu}$ has the additional weight

$$
q^{\left|\left\{r: i_{r}>j\right\}\right|-\left|\left\{r: i_{r}<i\right\}\right|}
$$

as a term of $\left(C_{[i j]}^{\ell}\right)_{i^{\prime} j^{\prime}}$. The proposition follows.

In what follows, the crucial observation is the following. Take $a_{\lambda, \mu}, \lambda=\lambda_{1} i j \lambda_{2}$, $\mu=\mu_{1} k l \mu_{2}, \lambda^{\prime}=\lambda_{1} j i \lambda_{2}, \mu^{\prime}=\mu_{1} l k \mu_{2}$ for $i<j$. Then

$$
q^{\text {inv } \mu-\operatorname{inv} \lambda} a_{\lambda, \mu}=q^{\text {inv } \mu^{\prime}-\operatorname{inv} \lambda^{\prime}} a_{\lambda^{\prime} \mu^{\prime}} \quad \bmod \mathcal{I}_{q-\mathrm{cf}},
$$

where $\mathcal{I}_{q-\text { cf }}$ is the ideal of $\mathcal{A}$ generated by the equations (7.1)-(7.3).

We show this by considering in turn each of the following possibilities: 
1. $i<j, k<l$

2. $i<j, k>l$

3. $i<j, k=l$

For example, to prove case (1), note that $a_{j l} a_{i k}-a_{i k} a_{j l}$ is a generator of $\mathcal{I}_{q-\mathrm{cf}}$, and that inv $\mu^{\prime}=\operatorname{inv} \mu+1$ and inv $\lambda^{\prime}=\operatorname{inv} \lambda+1$. Other cases are similarly straightforward.

Lemma 7.4 If $A$ is a q-Cartier-Foata matrix, $C$ is a q-right-quantum matrix.

Proof: We adapt the proof of Lemma 5.2. Choose $i, j, k>n, i<j$. The product $c_{i k} c_{j k}$ is the sum of terms of the form

$$
q^{-p-r} a_{i i_{1}} a_{i_{1} i_{2}} \cdots a_{i_{p} k} a_{j j_{1}} a_{j_{1} j_{2}} \cdots a_{j_{r} k}
$$

for $p, r \geq 0, i_{1}, \ldots, i_{p}, j_{1}, \ldots, j_{r} \leq n$.

Without changing the expression modulo $\mathcal{I}_{q-\text { cf }}$, we can repeat the procedure in the proof of Lemma 5.2, keeping track of weight changes. The resulting expression

$$
a_{j j_{1}^{\prime}} a_{j_{1}^{\prime} j_{2}^{\prime}} \cdots a_{j_{r^{\prime}}^{\prime} k} a_{i i_{1}^{\prime}} a_{i_{1}^{\prime} i_{2}^{\prime}} \cdots a_{i_{p^{\prime}}^{\prime} k}
$$

has, by the discussion preceding the lemma, weight $q^{-1-r^{\prime}-p^{\prime}}$ (the extra -1 comes from the fact that the step with starting height $j$ is now to the left of the step with starting height $i$ ), In other words,

$$
c_{j k} c_{i k}=q c_{i k} c_{j k}
$$

The proof of the other relation is completely analogous.

If $A$ is $q$-Cartier-Foata, Proposition 7.1 implies

$$
\left(I-A_{[n+1, n+1]}\right)_{n+1, n+1}^{-1}\left(I-\left(A^{n+1, n+1}\right)_{[n+2, n+2]}\right)_{n+2, n+2}^{-1} \cdots=\operatorname{det}_{q}^{-1}(I-A) \cdot \operatorname{det}_{q}\left(I-A_{0}\right)
$$

By Lemma 7.4, $C$ is $q$-right-quantum, so by Proposition 7.1

$$
\left(I-C_{[n+1, n+1]}\right)_{n+1, n+1}^{-1}\left(I-\left(C^{n+1, n+1}\right)_{[n+2, n+2]}\right)_{n+2, n+2}^{-1} \cdots=\operatorname{det}_{q}^{-1}(I-C),
$$

and hence

$$
\operatorname{det}_{q}{ }^{-1}\left(I-A_{0}\right) \cdot \operatorname{det}_{q}(I-A)=\operatorname{det}_{q}(I-C)
$$

The final step is to write entries of $C$ as quotients of quantum determinants.

Proposition 7.5 If $A$ is q-Cartier-Foata, then

$$
c_{i j}=-\operatorname{det}_{q}{ }^{-1}\left(I-A_{0}\right) \cdot \operatorname{det}_{q}\left(\begin{array}{cc}
I-A_{0} & -a_{* j} \\
-a_{i *} & -a_{i j}
\end{array}\right) .
$$


Proof: Again,

$$
\left(1-c_{i j}\right)^{-1}=\left(\left(I-\left(\begin{array}{cc}
q^{-1} A_{0} & q^{-1} a_{* j} \\
a_{i *} & a_{i j}
\end{array}\right)\right)^{-1}\right)_{i j},
$$

and because the matrix

$$
\left(\begin{array}{cc}
A_{0} & a_{* j} \\
a_{i *} & a_{i j}
\end{array}\right)
$$

is still $q$-Cartier-Foata, Proposition 7.1 shows that this is equal to

$$
\operatorname{det}_{q}^{-1}\left(I-\left(\begin{array}{cc}
A_{0} & a_{* j} \\
a_{i *} & a_{i j}
\end{array}\right)\right) \cdot \operatorname{det}_{q}\left(I-A_{0}\right) .
$$

The rest of the proof is exactly the same as in Proposition 5.3, with $\operatorname{det}_{q}$ playing the role of det.

We have proved the following.

Theorem 7.6 ( $q$-Cartier-Foata Sylvester's identity) Let $A=\left(a_{i j}\right)_{m \times m}$ be a qCartier-Foata matrix, and choose $n<m$. Let $A_{0}, a_{i *}, a_{* j}$ be defined as above, and let

$$
c_{i j}^{q}=-\operatorname{det}_{q}^{-1}\left(I-A_{0}\right) \cdot \operatorname{det}_{q}\left(\begin{array}{cc}
I-A_{0} & -a_{* j} \\
-a_{i *} & -a_{i j}
\end{array}\right), \quad C^{q}=\left(c_{i j}^{q}\right)_{n+1 \leq i, j \leq m} .
$$

Then

$$
\operatorname{det}_{q}^{-1}\left(I-A_{0}\right) \cdot \operatorname{det}_{q}(I-A)=\operatorname{det}_{q}\left(I-C^{q}\right) .
$$

\section{8 q-right-quantum analogue}

The results of the previous two sections easily extend to a $q$-right-quantum Sylvester's identity. Denote the ideal generated by relations $(7.4)-(7.5)$ by $\mathcal{I}_{q-\mathrm{rq}}$. It is easy to see that if $\lambda=\lambda_{1} i j \lambda_{2}, \mu=\mu_{1} k l \mu_{2}, \lambda^{\prime}=\lambda_{1} j i \lambda_{2}, \mu^{\prime}=\mu_{1} l k \mu_{2}$ and if $i<j$, then

$$
q^{\operatorname{inv} \mu-\operatorname{inv} \lambda} a_{\lambda, \mu}+q^{\operatorname{inv} \mu^{\prime}-\operatorname{inv} \lambda} a_{\lambda, \mu^{\prime}}=q^{\operatorname{inv} \mu-\operatorname{inv} \lambda^{\prime}} a_{\lambda^{\prime}, \mu}+q^{\operatorname{inv} \mu^{\prime}-\operatorname{inv} \lambda^{\prime}} a_{\lambda^{\prime}, \mu^{\prime}} \quad \bmod \mathcal{I}_{q-\mathrm{rq}} .
$$

Lemma 8.1 If $A$ is a q-right-quantum matrix, so is $C$.

Proof: This is a weighted analogue of Lemma 6.1. The sum over elements of $\mathcal{P}_{i j}^{k}\left(k_{1}, \ldots, k_{n}\right)$ with $a_{\lambda, \mu}$ weighted by $q^{\operatorname{inv} \mu-\operatorname{inv} \lambda}=q^{\text {inv } \mu}$ is modulo $\mathcal{I}_{q-\text { rq }}$ equal to both $c_{i k} c_{j k} S$ and $q^{-1} c_{j k} c_{i k} S$; this implies the relation (7.4) for elements of $C$, and the proof of (7.5) is completely analogous.

Proposition 8.2 If $A$ is q-right-quantum, then

$$
c_{i j}=-\operatorname{det}_{q}^{-1}\left(I-A_{0}\right) \cdot \operatorname{det}_{q}\left(\begin{array}{cc}
I-A_{0} & -a_{* j} \\
-a_{i *} & -a_{i j}
\end{array}\right) .
$$


Proof: The proof is exactly the same as the proof of Proposition 7.5.

Proposition 7.2, Lemma 8.1 and Proposition 8.2 imply the following theorem.

Theorem 8.3 (q-right-quantum Sylvester's identity) Let $A=\left(a_{i j}\right)_{m \times m}$ be a q-rightquantum matrix, and choose $n<m$. Let $A_{0}, a_{i *}, a_{* j}$ be defined as above, and let

$$
c_{i j}^{q}=-\operatorname{det}_{q}^{-1}\left(I-A_{0}\right) \cdot \operatorname{det}_{q}\left(\begin{array}{cc}
I-A_{0} & -a_{* j} \\
-a_{i *} & -a_{i j}
\end{array}\right), \quad C^{q}=\left(c_{i j}^{q}\right)_{n+1 \leq i, j \leq m} .
$$

Then

$$
\operatorname{det}_{q}^{-1}\left(I-A_{0}\right) \cdot \operatorname{det}_{q}(I-A)=\operatorname{det}_{q}\left(I-C^{q}\right) .
$$

\section{$9 q_{i j}$-Cartier-Foata analogue}

Now let us prove a multiparameter extension of Theorem 7.6. Choose $q_{i j} \neq 0$ for $i<j$, and recall that a matrix $A=\left(a_{i j}\right)_{m \times m}$ is q-Cartier-Foata if

$$
\begin{aligned}
q_{k l} a_{j l} a_{i k} & =q_{i j} a_{i k} a_{j l} \text { for } i<j, k<l, \\
a_{j l} a_{i k} & =q_{i j} q_{l k} a_{i k} a_{j l} \text { for } i<j, k>l, \\
a_{j k} a_{i k} & =q_{i j} a_{i k} a_{j k} \text { for } i<j,
\end{aligned}
$$

and q-right-quantum if

$$
\begin{aligned}
a_{j k} a_{i k} & =q_{i j} a_{i k} a_{j k} \text { for all } i<j, \\
a_{i k} a_{j l}-q_{i j}^{-1} a_{j k} a_{i l} & =q_{k l} q_{i j}^{-1} a_{j l} a_{i k}-q_{k l} a_{i l} a_{j k} \text { for all } i<j, k<l .
\end{aligned}
$$

Clearly, $q$-Cartier-Foata and $q$-right-quantum matrices are special cases of q-CartierFoata and q-right-quantum matrices, for $q_{i j}=q$ for all $i, j$. They were introduced in [KP] and were motivated by [M2].

If we define $q_{i i}=1$ and $q_{j i}=q_{i j}^{-1}$ for $i<j$, we can write the conditions (9.1)-(9.3) more concisely as

$$
q_{k l} a_{j l} a_{i k}=q_{i j} a_{i k} a_{j l}
$$

for all $i, j, k, l, i \neq j$, and (9.4)-(9.5) as

$$
a_{i k} a_{j l}-q_{i j}^{-1} a_{j k} a_{i l}=q_{k l} q_{i j}^{-1} a_{j l} a_{i k}-q_{k l} a_{i l} a_{j k}
$$

for all $i, j, k, l, i \neq j$.

In the following two sections, the weight $w(\lambda, \mu)$ is equal to

$$
\prod_{(i, j) \in \mathcal{I}(\mu)} q_{\mu_{j} \mu_{i}} \prod_{(i, j) \in \mathcal{I}(\lambda)} q_{\lambda_{j} \lambda_{i}}^{-1}
$$


For example,

$$
\operatorname{det}_{q}(I-A)=\sum_{J \subseteq[m]}(-1)^{|J|} \operatorname{det}_{\mathbf{q}} A_{J}
$$

where

$$
\operatorname{det}_{q} A_{J}=\operatorname{det}_{q}\left(a_{i j}\right)_{i, j \in J}=\sum_{\sigma \in S_{J}}\left(\prod_{p<q: \sigma(p)>\sigma(q)} q_{\sigma(q) \sigma(p)}^{-1}\right) a_{\sigma\left(j_{1}\right) j_{1}} \cdots a_{\sigma\left(j_{k}\right) j_{k}}
$$

for $J=\left\{j_{1}<j_{2}<\ldots<j_{k}\right\}$.

The following extends Proposition 7.1. A special case (when $i=j=1$ ) is [KP, Proposition 7.3, Proposition 8.1]. The proof in this more general case is almost exactly the same and we omit it.

Proposition 9.1 If $A=\left(a_{i j}\right)_{m \times m}$ is a $\mathbf{q}$-Cartier-Foata matrix or a $\mathbf{q}$-right-quantum matrix, we have

$$
\left(\frac{1}{I-A_{[i j]}}\right)_{i j}=(-1)^{i+j} \frac{1}{\operatorname{det}_{\mathbf{q}}(I-A)} \cdot \operatorname{det}_{\mathbf{q}}(I-A)^{j i}
$$

for all $i, j$, where

$$
A_{[i j]}=\left(\begin{array}{cccccc}
q_{1 i}^{-1} a_{11} & \cdots & q_{1 i}^{-1} a_{1 j} & q_{1 i}^{-1} q_{j, j+1} a_{1, j+1} & \cdots & q_{1 i}^{-1} q_{j m} a_{1 m} \\
\vdots & \ddots & \vdots & \vdots & \ddots & \vdots \\
q_{i-1, i}^{-1} a_{i-1,1} & \cdots & q_{i-1, i}^{-1} a_{i-1, j} & q_{i-1, i}^{-1} q_{j, j+1} a_{i-1, j+1} & \cdots & q_{i-1, i}^{-1} q_{j m} a_{i-1, m} \\
a_{i 1} & \cdots & a_{i j} & q_{j, j+1} a_{i, j+1} & \cdots & q_{j m} a_{i, m} \\
\vdots & \ddots & \vdots & \vdots & \ddots & \vdots \\
a_{m 1} & \cdots & a_{m j} & q_{j, j+1} a_{m, j+1} & \cdots & q_{j m} a_{m m}
\end{array}\right) .
$$

Assume that $q_{i j}=q_{i^{\prime} j^{\prime}}$ for $i, i^{\prime} \leq n, j, j^{\prime}>n$; denote this value by $q$. We use Theorem 3.1 for the matrix $A_{[i j]}$ and the corresponding $C=\left(c_{i^{\prime} j^{\prime}}^{\prime}\right)_{n+1 \leq i^{\prime}, j^{\prime} \leq m}$. Define

$$
c_{i^{\prime} j^{\prime}}=a_{i^{\prime} j^{\prime}}+q^{-1} a_{i^{\prime} *}\left(I-q^{-1} A_{0}\right)^{-1} a_{* j^{\prime}}
$$

for $i^{\prime}, j^{\prime}>n$. If $i^{\prime}<i, j^{\prime} \leq j$, we have

$$
c_{i^{\prime} j^{\prime}}^{\prime}=q_{i^{\prime} i}^{-1} a_{i^{\prime} j^{\prime}}+\left(q_{i^{\prime} i}^{-1} a_{i^{\prime} *}\right)\left(I-q^{-1} A_{0}\right)^{-1}\left(q^{-1} a_{* j^{\prime}}\right)=q_{i^{\prime} i}^{-1} c_{i^{\prime} j^{\prime}}
$$

if $i^{\prime}<i, j^{\prime}>j$, we have

$$
c_{i^{\prime} j^{\prime}}^{\prime}=q_{i^{\prime} i}^{-1} q_{j j^{\prime}} a_{i^{\prime} j^{\prime}}+\left(q_{i^{\prime} i}^{-1} a_{i^{\prime} *}\right)\left(I-q^{-1} A_{0}\right)^{-1}\left(q^{-1} q_{j j^{\prime}} a_{* j^{\prime}}\right)=q_{i^{\prime} i}^{-1} q_{j j^{\prime}} c_{i^{\prime} j^{\prime}} ;
$$

if $i^{\prime} \geq i, j^{\prime} \leq j$, we have

$$
c_{i^{\prime} j^{\prime}}^{\prime}=a_{i^{\prime} j^{\prime}}+a_{i^{\prime} *}\left(I-q^{-1} A_{0}\right)^{-1}\left(q^{-1} a_{* j^{\prime}}\right)=c_{i^{\prime} j^{\prime}} ;
$$

and if $i^{\prime} \geq i, j^{\prime}>j$, we have

$$
c_{i^{\prime} j^{\prime}}^{\prime}=q_{j j^{\prime}} a_{i^{\prime} j^{\prime}}+a_{i^{\prime} *}\left(I-q^{-1} A_{0}\right)^{-1}\left(q^{-1} q_{j j^{\prime}} a_{* j^{\prime}}\right)=q_{j j^{\prime}} C_{i^{\prime} j^{\prime}} .
$$

We have proved the following. 
Proposition 9.2 With $A_{[i j]}$ as defined above and with $C=\left(c_{i^{\prime} j^{\prime}}\right)_{n+1 \leq i^{\prime}, j^{\prime} \leq m}$ for

$$
c_{i^{\prime} j^{\prime}}=a_{i^{\prime} j^{\prime}}+a_{i^{\prime} *}\left(I-q^{-1} A_{0}\right)^{-1}\left(q^{-1} a_{* j^{\prime}}\right),
$$

we have

$$
\left(I-A_{[i j]}\right)_{i^{\prime} j^{\prime}}^{-1}=\left(I-C_{[i j]}\right)_{i^{\prime} j^{\prime}}^{-1}
$$

REMARK 9.3 Another way to characterize $A_{[i j]}$ is to say that the entry $a_{k l}$ has weight

$$
\left\{\begin{array} { c } 
{ q _ { j l } : l > j } \\
{ 1 : l \leq j }
\end{array} \cdot \left\{\begin{array}{c}
q_{k i}^{-1}: k<i \\
1: k \geq i
\end{array}\right.\right.
$$

That means that in $\left(A_{[i j]}^{\ell}\right)_{i_{1} i_{\ell}}$,

$$
a_{i_{1} i_{2}} a_{i_{2} i_{3}} \cdots a_{i_{\ell-1} i_{\ell}}
$$

has weight

$$
\prod_{i_{r}>j} q_{j i_{r}} \cdot \prod_{i_{r}<i} q_{i_{r} i}^{-1}
$$

An alternative way to prove the proposition is analogous to the proof of Proposition 7.2 outlined in Remark 7.3.

$$
\text { If } a_{\lambda, \mu}, \lambda=\lambda_{1} i j \lambda_{2}, \mu=\mu_{1} k l \mu_{2}, \lambda^{\prime}=\lambda_{1} j i \lambda_{2}, \mu^{\prime}=\mu_{1} l k \mu_{2} \text { and if } i<j \text {, then }
$$

$$
\left(\prod_{(i, j) \in \mathcal{I}(\mu)} q_{\mu_{j} \mu_{i}} \prod_{(i, j) \in \mathcal{I}(\lambda)} q_{\lambda_{j} \lambda_{i}}^{-1}\right) a_{\lambda, \mu}=\left(\prod_{(i, j) \in I\left(\mu^{\prime}\right)} q_{\mu_{j}^{\prime} \mu_{i}^{\prime}} \prod_{(i, j) \in I\left(\lambda^{\prime}\right)} q_{\lambda_{j}^{\prime} \lambda_{i}^{\prime}}^{-1}\right) a_{\lambda^{\prime} \mu^{\prime}} \quad \bmod \mathcal{I}_{q-\mathrm{cf}}
$$

where $\mathcal{I}_{\mathbf{q}-\mathrm{cf}}$ is the ideal of $\mathcal{A}$ generated by the equations (9.1)-(9.3).

As in the $q$-Cartier-Foata case, we show this by considering in turn each of the possibilities $k<l, k>l, k=l$.

Lemma 9.4 If $A$ is a $\mathbf{q}$-Cartier-Foata matrix, $C$ is a $\mathbf{q}$-right-quantum matrix.

Proof: We adapt the proof of Lemma 7.4. Choose $i, j, k>n, i<j$. The product $c_{i k} c_{j k}$ is the sum of terms of the form

$$
q^{-p-r} a_{i i_{1}} a_{i_{1} i_{2}} \cdots a_{i_{p} k} a_{j j_{1}} a_{j_{1} j_{2}} \cdots a_{j_{r} k}
$$

for $p, r \geq 0, i_{1}, \ldots, i_{p}, j_{1}, \ldots, j_{r} \leq n$.

Note that since

$$
q^{-p-r}=q_{j_{1} k} \cdots q_{j_{r} k} q_{i_{1} i}^{-1} \cdots q_{i_{p} i}^{-1} q_{j_{1} i}^{-1} \cdots q_{j_{r} i}^{-1} q_{j_{1} j}^{-1} \cdots q_{j_{r} j}^{-1},
$$

the weight of $a_{i i_{1}} a_{i_{1} i_{2}} \cdots a_{i_{p} k} a_{j j_{1}} a_{j_{1} j_{2}} \cdots a_{j_{r} k}$ is of the form

$$
\prod_{(i, j) \in \mathcal{I}(\mu)} q_{\mu_{j} \mu_{i}} \prod_{(i, j) \in \mathcal{I}(\lambda)} q_{\lambda_{j} \lambda_{i}}^{-1}
$$


for $\lambda=i i_{1} \ldots i_{p} j j_{1} \ldots j_{r}$ and $\mu=i_{1} \ldots i_{p} k j_{1} \ldots j_{r} k$. Without changing the expression modulo $\mathcal{I}_{\mathbf{q}-\mathrm{cf}}$, we can repeat the procedure in the proof of Lemma 5.2, but changing the weight at each switch. The resulting expression

$$
a_{j j_{1}^{\prime}} a_{j_{1}^{\prime} j_{2}^{\prime}} \cdots a_{j_{r^{\prime}}^{\prime} k} a_{i i_{1}^{\prime}} a_{i_{1}^{\prime} i_{2}^{\prime}} \cdots a_{i_{p^{\prime}}^{\prime} k}
$$

has, by the discussion preceding the lemma, weight

$$
q_{i_{1}^{\prime} k} \cdots q_{i_{p^{\prime}}^{\prime} k} q_{j_{1}^{\prime} j}^{-1} \cdots q_{j_{r^{\prime} j}^{\prime} j}^{-1} q_{i_{1}^{\prime} j}^{-1} \cdots q_{i_{p^{\prime}}^{\prime} j}^{-1} q_{i_{1}^{\prime} i}^{-1} \cdots q_{i_{p^{\prime}}^{\prime}}^{-1} q_{i j}^{-1}=q^{-r^{\prime}-p^{\prime}} q_{i j}^{-1}
$$

(the extra $q_{i j}^{-1}$ comes from the fact that the step with starting height $j$ is now to the left of the step with starting height $i$ ), In other words,

$$
c_{j k} c_{i k}=q_{i j} c_{i k} c_{j k}
$$

The proof of the other relation is completely analogous.

If $A$ is q-Cartier-Foata, Proposition 9.1 implies

$$
\left(I-A_{[n+1, n+1]}\right)_{n+1, n+1}^{-1}\left(I-\left(A^{n+1, n+1}\right)_{[n+2, n+2]}\right)_{n+2, n+2}^{-1} \cdots=\operatorname{det}_{\mathbf{q}}^{-1}(I-A) \cdot \operatorname{det}_{\mathbf{q}}\left(I-A_{0}\right) .
$$

By Lemma 7.4, $C$ is q-right-quantum, so by Proposition 9.1

$$
\left(I-C_{[n+1, n+1]}\right)_{n+1, n+1}^{-1}\left(I-\left(C^{n+1, n+1}\right)_{[n+2, n+2]}\right)_{n+2, n+2}^{-1} \cdots=\operatorname{det}_{\mathbf{q}}^{-1}(I-C),
$$

and hence

$$
\operatorname{det}_{\mathbf{q}}^{-1}\left(I-A_{0}\right) \cdot \operatorname{det}_{\mathbf{q}}(I-A)=\operatorname{det}_{\mathbf{q}}(I-C)
$$

So far, the extension to the multiparameter case has been straightforward. However, we need something extra for the proof of the analogue of Proposition 7.5 since the matrix

$$
\left(\begin{array}{cc}
A_{0} & a_{* j} \\
a_{i *} & a_{i j}
\end{array}\right)
$$

is in general not q-Cartier-Foata. However, a special case of the first statement of Proposition 9.1 holds under slightly weaker conditions.

Proposition 9.5 Assume that for $A=\left(a_{i j}\right)_{m \times m}$, the submatrix $\left(a_{i j}\right)_{m \times(m-1)}$ is a qCartier-Foata or a $\mathbf{q}$-right-quantum matrix. Then

$$
\left(\frac{1}{I-A_{[m m]}}\right)_{m m}=\frac{1}{\operatorname{det}_{\mathbf{q}}(I-A)} \cdot \operatorname{det}_{\mathbf{q}}\left(I-A^{m m}\right),
$$

where $A_{[m m]}$ is defined as in Proposition 9.1.

Proof: When $i=j=m$, the relations (9.1)-(9.5) for $k=m$ or $l=m$ are never used in the proof of Proposition 9.1. 
In other words, even though only the first $n$ columns of

$$
\left(\begin{array}{cc}
A_{0} & a_{* j} \\
a_{i *} & a_{i j}
\end{array}\right)
$$

satisfy the q-Cartier-Foata condition, we still have

$$
\begin{gathered}
\left(1-c_{i j}\right)^{-1}=\left(\left(I-\left(\begin{array}{cc}
q^{-1} A_{0} & q^{-1} a_{* j} \\
a_{i *} & a_{i j}
\end{array}\right)\right)^{-1}\right)_{i j}= \\
=\operatorname{det}_{\mathbf{q}}^{-1}\left(I-\left(\begin{array}{cc}
A_{0} & a_{* j} \\
a_{i *} & a_{i j}
\end{array}\right)\right) \cdot \operatorname{det}_{\mathbf{q}}\left(I-A_{0}\right) .
\end{gathered}
$$

Proposition 9.6 If $A$ is $\mathbf{q}$-Cartier-Foata, then

$$
c_{i j}=-\operatorname{det}_{\mathbf{q}}^{-1}\left(I-A_{0}\right) \cdot \operatorname{det}_{\mathbf{q}}\left(\begin{array}{cc}
I-A_{0} & -a_{* j} \\
-a_{i *} & -a_{i j}
\end{array}\right) .
$$

Proof: This follows from the previous proposition, using the same technique as in the proof of Proposition 5.3.

We have proved the following.

Theorem 9.7 (q-Cartier-Foata Sylvester's theorem) Let $A=\left(a_{i j}\right)_{m \times m}$ be a qCartier-Foata matrix, and choose $n<m$. Let $A_{0}, a_{i *}, a_{* j}$ be defined as above, and let

$$
c_{i j}^{\mathbf{q}}=-\operatorname{det}_{\mathbf{q}}^{-1}\left(I-A_{0}\right) \cdot \operatorname{det}_{\mathbf{q}}\left(\begin{array}{cc}
I-A_{0} & -a_{* j} \\
-a_{i *} & -a_{i j}
\end{array}\right), \quad C^{\mathbf{q}}=\left(c_{i j}^{\mathbf{q}}\right)_{n+1 \leq i, j \leq m} .
$$

Suppose $q_{i j}=q_{i^{\prime} j^{\prime}}$ for all $i, i^{\prime} \leq n$ and $j, j^{\prime}>n$. Then

$$
\operatorname{det}_{\mathbf{q}}^{-1}\left(I-A_{0}\right) \cdot \operatorname{det}_{\mathbf{q}}(I-A)=\operatorname{det}_{\mathbf{q}}\left(I-C^{\mathbf{q}}\right) .
$$

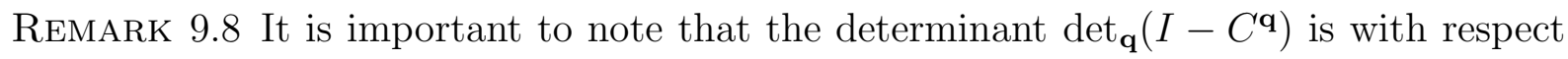
to $\mathcal{C}$, the algebra generated by $c_{i j}$ 's, not with respect to $\mathcal{A}$. For example, for $n=2$ and $m=4$, we have

$$
\operatorname{det}_{\mathbf{q}}\left(I-C^{\mathbf{q}}\right)=1-c_{33}^{\mathbf{q}}-c_{44}^{\mathbf{q}}+c_{33}^{\mathbf{q}} c_{44}^{\mathbf{q}}-q_{34}^{-1} c_{43}^{\mathbf{q}} c_{34}^{\mathbf{q}} .
$$

REMARK 9.9 The condition $q_{i j}=q_{i^{\prime} j^{\prime}}$ whenever $i, i^{\prime} \leq n, j, j^{\prime}>n$ is indeed necessary, as shown by the following. Take $n=1$ and $m=3$. In $\operatorname{det}_{\mathbf{q}}^{-1}\left(I-A_{0}\right) \cdot \operatorname{det}_{\mathbf{q}}(I-A)$ we have the term

$$
-q_{12}^{-1} q_{13}^{-1} a_{21} a_{32} a_{13}
$$

while in $\operatorname{det}_{\mathbf{q}}\left(I-C^{\mathbf{q}}\right)$ we have

$$
-q_{23}^{-1}\left(-a_{32}\right)\left(-q_{12}^{-1} a_{21} a_{13}\right)=-q_{12}^{-2} a_{21} a_{32} a_{13} .
$$




\section{$10 q_{i j}$-right-quantum analogue}

The results in this section are almost complete copies of proofs above.

Assume we have a q-right-quantum matrix, with $q_{i j}=q$ for $i \leq n, j>n$. In the notation of the previous section, we have the following.

Lemma 10.1 If $A$ is a q-Cartier-Foata matrix, $C$ is a q-right-quantum matrix.

Proof: We use a combination of proofs of Lemmas 8.1 and 9.4.

Proposition 10.2 If $A$ is $\mathbf{q}$-right-quantum, then

$$
c_{i j}=-\operatorname{det}_{\mathbf{q}}^{-1}\left(I-A_{0}\right) \cdot \operatorname{det}_{\mathbf{q}}\left(\begin{array}{cc}
I-A_{0} & -a_{* j} \\
-a_{i *} & -a_{i j}
\end{array}\right) .
$$

Proof: We use the same technique as in the proof of Proposition 9.6.

This finishes the proof of Theorem 1.4.

\section{The $\beta$-extension}

Theorem 1.1 trivially implies that

$$
(\operatorname{det} B)^{\beta}=(\operatorname{det} A)^{\beta} \cdot\left(\operatorname{det} A_{0}\right)^{\beta(m-n-1)}
$$

for any $\beta \in \mathbb{Z}$, where $a_{i j}$ are commutative variables and

$$
b_{i j}=\operatorname{det}\left(\begin{array}{cc}
A_{0} & a_{* j} \\
a_{i *} & a_{i j}
\end{array}\right), \quad B=\left(b_{i j}\right)_{n+1 \leq i, j \leq m} .
$$

It is not immediately clear what the non-commutative extension of this could be. Of course, Theorem 5.4 implies that

$$
(\operatorname{det}(I-C))^{\beta}=\left(\operatorname{det}^{-1}\left(I-A_{0}\right) \cdot \operatorname{det}(I-A)\right)^{\beta}
$$

for

$$
c_{i j}=-\operatorname{det}^{-1}\left(I-A_{0}\right) \cdot \operatorname{det}\left(\begin{array}{cc}
I-A_{0} & -a_{* j} \\
-a_{i *} & -a_{i j}
\end{array}\right), \quad C=\left(c_{i j}\right)_{n+1 \leq i, j \leq m},
$$

where $A$ is a Cartier-Foata or right-quantum matrix, but this does not tell us how to calculate terms of $(\operatorname{det}(I-C))^{\beta}$. However, a technique similar to the proof of the $\beta$-extension of the non-commutative MacMahon Master Theorem, [KP, §10], gives a reasonable interpretation of $(\operatorname{det}(I-C))^{\beta}$ for $\beta \in \mathbb{Z}$ when $A$ is a Cartier-Foata matrix.

We need some terminology from $[\mathrm{KP}]$. A balanced sequence (b-sequence) is a finite sequence of steps such that the number of steps starting at height $i$ is equal to the number of steps ending at height $i$, for all $i$. We denote this number by $k_{i}$, and call $\left(k_{1}, \ldots, k_{m}\right)$ the 
type of the b-sequence. An ordered sequence (o-sequence) is a b-sequence where the steps starting at smaller height always precede steps starting at larger heights. In other words, an o-sequence of type $\left(k_{1}, \ldots, k_{m}\right)$ is a sequence of $k_{1}$ steps starting at height 1 , then $k_{2}$ steps starting at height 2 , etc., so that $k_{i}$ steps end at height $i$. Denote by $\mathbf{O}\left(k_{1}, \ldots, k_{m}\right)$ the set of all o-sequences of type $\left(k_{1}, \ldots, k_{m}\right)$. Finally, consider a lattice path from $(0,1)$ to $\left(x_{1}, 1\right)$ that never goes below $y=1$ or above $y=m$, then a lattice path from $\left(x_{1}, 2\right)$ to $\left(x_{2}, 2\right)$ that never goes below $y=2$ or above $y=m$, etc.; in the end, take a straight path from $\left(x_{m-1}, m\right)$ to $\left(x_{m}, m\right)$. We call this a path sequence ( $p$-sequence). Observe that every p-sequence is also a b-sequence. Denote by $\mathbf{P}\left(k_{1}, \ldots, k_{m}\right)$ the set of all p-sequences of type $\left(k_{1}, \ldots, k_{m}\right)$.

In $[\mathrm{KP}, \S 2]$ a bijection

$$
\varphi: \mathbf{O}\left(k_{1}, \ldots, k_{m}\right) \longrightarrow \mathbf{P}\left(k_{1}, \ldots, k_{m}\right)
$$

was defined (which proved various forms of the MacMahon Master Theorem) as follows. Take an o-sequence $\alpha$, and let $[0, x]$ be the maximal interval on which it is part of a psequence, i.e. the maximal interval $[0, x]$ on which the o-sequence has the property that if a step ends at level $i$, and the following step starts at level $j>i$, the o-sequence stays on or above height $j$ afterwards. Let $i$ be the height at $x$. Choose the step $\left(x^{\prime}, i\right) \rightarrow\left(x^{\prime}+1, i^{\prime}\right)$ in the o-sequence that is the first to the right of $x$ that starts at level $i$ (such a step exists because an o-sequence is a balanced sequence). Continue switching this step with the one to the left until it becomes the step $(x, i) \rightarrow\left(x+1, i^{\prime}\right)$. The new object is part of a $\mathrm{p}-$ sequence at least on the interval $[0, x+1]$. Continuing this procedure we get a p-sequence $\varphi(\alpha)$.

A lattice path from $i$ to $i$ with each height appearing at most once as the starting height is called a disjoint cycle.

For an o-sequence $a_{\lambda, \mu}$, take the corresponding p-sequence $a_{\lambda^{\prime}, \mu^{\prime}}=\varphi\left(a_{\lambda, \mu}\right)$. If the first repeated height in $a_{\lambda^{\prime}, \mu^{\prime}}$ is the starting height of the sequence, the sequence starts with a disjoint cycle; remove it and repeat the algorithm. If the first repeated height in $a_{\lambda^{\prime}, \mu^{\prime}}$ is not the starting height of the sequence, we have $\lambda^{\prime}$ starting with $i_{1} i_{2} \cdots i_{p} i_{p+1} i_{p+2} \cdots i_{p+r-1}$ and $\mu^{\prime}$ starting with $i_{2} i_{3} \cdots i_{p+1} i_{p+2} \cdots i_{p}$ for different indices $i_{1}, \ldots, i_{p+r-1}$. Then we can move the disjoint cycle $i_{p} \rightarrow i_{p+1} \rightarrow \ldots \rightarrow i_{p+r-1} \rightarrow i_{p}$ to the beginning, remove it, and repeat the algorithm with the rest of the sequence. The resulting sequence is a concatenation of disjoint cycles, and we call it the disjoint cycle decomposition of the osequence $a_{\lambda, \mu}$ (or of the p-sequence $a_{\lambda^{\prime}, \mu^{\prime}}$ ). For example, the disjoint cycle decomposition of

$$
a_{13} a_{11} a_{12} a_{13} a_{22} a_{23} a_{22} a_{21} a_{23} a_{22} a_{23} a_{32} a_{31} a_{31} a_{33} a_{32} a_{32} a_{33} a_{33}
$$

is

$$
a_{22} a_{32} a_{23} a_{13} a_{31} a_{11} a_{22} a_{12} a_{21} a_{13} a_{31} a_{33} a_{23} a_{32} a_{22} a_{23} a_{32} a_{33} a_{33} .
$$

We say that two cycles in the disjoint cycle decomposition are disjoint if the sets of their starting heights are disjoint. 
Recall that for a Cartier-Foata matrix $A$, the matrix $C=\left(c_{i j}\right)_{n+1 \leq i, j \leq m}$ with

$$
c_{i j}=-\operatorname{det}^{-1}\left(I-A_{0}\right) \cdot \operatorname{det}\left(\begin{array}{cc}
I-A_{0} & -a_{* j} \\
-a_{i *} & -a_{i j}
\end{array}\right)
$$

is right-quantum by Lemma 5.2 and Proposition 5.3, so

$$
\begin{gathered}
\operatorname{det}^{-1}(I-C)=(I-C)_{n+1, n+1}^{-1}\left(I-C^{n+1, n+1}\right)_{n+2, n+2}^{-1} \cdots= \\
=(I-A)_{n+1, n+1}^{-1}\left(I-A^{n+1, n+1}\right)_{n+2, n+2}^{-1} \cdots
\end{gathered}
$$

by Theorem 3.1. The last expression is the sum over all sequences which are concatenations of a lattice path from $n+1$ to $n+1$, a lattice path from $n+2$ to $n+2$, etc.

Theorem 11.1 ( $\beta$-extension of Cartier-Foata Sylvester's identity) Assume $A=$ $\left(a_{i j}\right)_{m \times m}$ is a Cartier-Foata matrix. For

$$
C=\left(c_{i j}\right)_{n+1 \leq i, j \leq m} \quad \text { with } \quad c_{i j}=-\operatorname{det}^{-1}\left(I-A_{0}\right) \cdot \operatorname{det}\left(\begin{array}{cc}
I-A_{0} & -a_{* j} \\
-a_{i *} & -a_{i j}
\end{array}\right) .
$$

For each $\beta \in \mathbb{Z}$, the expression

$$
\left(\frac{1}{\operatorname{det}(I-C)}\right)^{\beta}
$$

is equal to

$$
\sum e_{\mu}(\beta) a_{\lambda, \mu}
$$

where $\mu$ runs over all words in the alphabet $\{1, \ldots, m\}, \lambda$ is the non-decreasing rearrangement of $\mu$, and $e_{\mu}(\beta)$ is a polynomial function of $\beta$ that is calculated as follows. Let $u_{1} u_{2} \ldots u_{k}$ be the disjoint cycle decomposition of $a_{\lambda, \mu}$. Let $\mathcal{J}$ be the set of $i \in\{1, \ldots, k\}$ such that $u_{i}$ contains a height $>n$. Then

$$
e_{\mu}(\beta)=\sum_{\pi}\left(\begin{array}{c}
\beta+l-1-d(\pi) \\
l
\end{array}\right),
$$

where the sum is over all permutations $\pi \in S_{k}$ with the following properties:

- if $i<j, \pi(i)>\pi(j)$, then $u_{\pi(i)}, u_{\pi(j)}$ are disjoint;

- for each $i \notin \mathcal{J}$ there exists $j>i$ such that $u_{\pi(i)}$ and $u_{\pi(j)}$ are not disjoint;

- if $\pi(i)>\pi(i+1)$ then $\pi(i) \in \mathcal{J}$.

Here $d(\pi)$ denotes the number of descents of the subword of $(\pi(1), \pi(2), \ldots, \pi(k))$ composed of $\pi(i) \in \mathcal{J}$, and $l=|\mathcal{J}|$. 
EXAMPLE 11.2 Take $\mu=132521421325$. The disjoint cycle decomposition of the osequence $a_{11} a_{13} a_{12} a_{25} a_{22} a_{21} a_{24} a_{32} a_{31} a_{43} a_{52} a_{55}$ is

$$
u_{1} u_{2} u_{3} u_{4} u_{5} u_{6}=\left(a_{11}\right)\left(a_{25} a_{52}\right)\left(a_{22}\right)\left(a_{13} a_{32} a_{21}\right)\left(a_{12} a_{24} a_{43} a_{31}\right)\left(a_{55}\right) .
$$

We have $\mathcal{J}=\{2,4,5,6\}$, the only permutations in $S_{6}$ that appear in the sum (11.2) are $213456,213465,261345$ with $d(213456)=0, d(213465)=1, d(261345)=1$. Therefore

$$
e_{\mu}(\beta)=\left(\begin{array}{c}
\beta+3 \\
4
\end{array}\right)+2\left(\begin{array}{c}
\beta+2 \\
4
\end{array}\right)=\frac{\beta^{4}}{8}+\frac{5 \beta^{3}}{12}+\frac{3 \beta^{2}}{8}+\frac{\beta}{12} .
$$

EXAMPLE 11.3 Take $n=0$. In this case $\mathcal{J}=\{1, \ldots, k\}$, only the first condition is not vacuously true on $\pi$, and we get the $\beta$-extension of MacMahon Master Theorem, [KP, Theorem 10.5].

It is clear that each term of $(\operatorname{det}(I-C))^{-\beta}$ is an o-sequence modulo $\mathcal{I}_{\text {cf }}$, and that the coefficients of o-sequences are polynomial functions of $\beta$. Therefore it is enough to prove the theorem for $\beta \in \mathbb{N}$, and this is an enumerative problem. We are given an o-sequence $a_{\lambda, \mu}$ and $\beta$ slots, and we have to calculate in how many ways we can choose terms of $(\operatorname{det}(I-C))^{-1}$ in each slot so that their product is, modulo $\mathcal{I}_{\text {cf }}$, equal to $a_{\lambda, \mu}$.

We start the proof with a lemma.

Lemma 11.4 All the steps in a cycle of the disjoint cycle decomposition must be placed in the same slot.

Proof: This is proved in exactly the same way as the proof of [KP, Lemma 10.4], since all we used there was that the sequence chosen in each slot must be balanced, which is also true in our case.

Proof of Theorem 11.1. We call cycles with all heights $\leq n$ low cycles, and cycles containing at least one height $>n$ high cycles.

The lemma tells us that we must choose a permutation $\pi \in S_{k}$ such that $u_{1} \cdots u_{k}=$ $u_{\pi(1)} \cdots u_{\pi(k)}$ modulo $\mathcal{I}_{\text {cf }}$, and place the cycles $u_{\pi(1)}, \ldots, u_{\pi(k)}$ in the $\beta$ slots so that the cycles in each slot give a term appearing in $(\operatorname{det}(I-C))^{-1}$.

Two cycles commute if and only if they are disjoint. That means that the condition $u_{1} \cdots u_{k}=u_{\pi(1)} \cdots u_{\pi(k)}$ is equivalent to

- if $i<j, \pi(i)>\pi(j)$, then $u_{\pi(i)}, u_{\pi(j)}$ are disjoint,

which is the first condition in Theorem 11.1.

Take a low cycle $u_{\pi(i)}$, and assume that it is disjoint with all $u_{\pi(j)}$ for $j>i$. That means we can push it to the end of the chosen slot without changing the sequence modulo $\mathcal{I}_{\text {cf }}$. But then the sequence in the slot is not equal to a sequence of the form (11.1) modulo $\mathcal{I}_{\text {cf. }}$. Therefore

- for each $i \notin \mathcal{J}$ there exists $j>i$ such that $u_{\pi(i)}$ and $u_{\pi(j)}$ are not disjoint, 
which is the second condition in Theorem 11.1.

Finally, assume that we have $\pi(i)>\pi(i+1)$ with $\pi(i) \notin \mathcal{J}$. Then $\pi(i)$ must be placed in the same slot as a high cycle $\pi(j)$ for $j>i$, and so $\pi(i+1)$, which commutes with $\pi(i)$, must be placed in the same slot as well. But then this placement of cycles in slots is already counted in the permutation where $\pi(i)$ and $\pi(i+1)$ are switched. Therefore we have

$$
\text { - if } \pi(i)>\pi(i+1) \text { then } \pi(i) \in \mathcal{J} \text {, }
$$

which is the third condition in Theorem 11.1.

We have described all permutations that give $a_{\lambda, \mu}$, and now we have to find the number of ways to place $u_{\pi(1)}, \ldots, u_{\pi(k)}$ in the $\beta$ slots so that the cycles in each slot give a term appearing in $(\operatorname{det}(I-C))^{-1}$. All cycles between two consecutive high cycles must appear in the same slot as the right-hand high cycle. Therefore placing the cycles in slots is the same as placing $\beta-1$ dividers after (some of the) high cycles. Of course, there are $\left(\begin{array}{c}\beta-1+l \\ l\end{array}\right)$ ways of doing this, but we can get the same terms several times: if we take two consecutive high cycles $u_{\pi(i)}, u_{\pi(j)}$ with $i<j, \pi(i)>\pi(j)$, then $u_{\pi(i)}$ must necessarily commute with $u_{\pi(j)}$ and with all the low cycles between them, we can move $u_{\pi(j)}$ to the right of $u_{\pi(i)}$, possibly move some of the low cycles before $u_{\pi(j)}$ to the right of $u_{\pi(i)}$, and we see that this term has already been counted for a different $\pi$. In order to avoid overcounting, we have to place a divider after $u_{\pi(i)}$. Therefore the number of unique placements in slots corresponding to $\pi$ is $\left(\begin{array}{c}\beta-1+l-d(\pi) \\ l\end{array}\right)$, and this finishes the proof of Theorem 11.1.

Acknowledgments. The author is grateful to Igor Pak for numerous suggestions, and to Pavel Etingof and Alexander Molev for help with the references.

\section{References}

[AAM] A. G. Akritas, E. K. Akritas, G. I. Malaschonok, Various proofs of Sylvester's (determinant) identity, Math. Comput. Simulation 42 (1996), 585-593

[B] E. H. Bareiss, Sylvester's identity and multistep integer-preserving Gaussian elimination, Math. Comput. 22 (1968), 565-578

[CF] P. Cartier and D. Foata, Problèmes combinatoires de commutation et réarrangements, Lecture Notes in Mathematics, No. 85, Springer, Berlin, 1969

[EP] P. Etingof and I. Pak, An algebraic extension of the MacMahon Master Theorem, preprint (2006)

[ER] P. Etingof and V. Retakh, Quantum determinants and quasideterminants, Asian J. Math. 3 (1999), 345-351

[F1] D. Foata, Étude algébrique de certains problèmes d'analyse combinatoire et du calcul des probabilités, Publ. Inst. Statist. Univ. Paris 14 (1965), 81-241 
[F2] D. Foata, A Noncommutative Version of the Matrix Inversion Formula, Adv. Math. 31 (1979), 330-349

[GLZ] S. Garoufalidis, T. Tq Lê and D. Zeilberger, The Quantum MacMahon Master Theorem, arXive:math.QA/0303319, to appear in Proc. Natl. Acad. of Sci.

[GeR] I. M. Gelfand and V. S. Retakh, Determinants of matrices over noncommutative rings, Funct. Anal. Appl. 25 (1991), no. 2, 91-102

[GeR] I. M. Gelfand and V. S. Retakh, A theory of non-commutative determinants and characteristic functions of graphs, Funct. Anal. Appl. 26 (1992), no. 4, $1-20$

[GGRW] I. Gelfand, S. Gelfand, V. Retakh and R. L. Wilson, Quasideterminants, Adv. Math. 193 (2005), 56-141

[HM] M. J. Hopkins and A. I. Molev, A $q$-analogue of the centralizer construction and skew representations of the quantum affine algebra, arXive:QA/0606121

[K] C. Kassel, Quantum groups, Graduate Texts in Mathematics 155, SpringerVerlag (1995)

[KP] M. Konvalinka and I. Pak, Non-commutative extensions of MacMahon's Master Theorem, arXive:math.CO/0607737, to appear in Adv. Math.

[KL] D. Krob, B. Leclerc, Minor Identities for Quasi-Determinants and Quantum Determinants, Commun. Math. Phys. 169 (1995), 1-23

[M1] Yu. I. Manin, Some remarks on Koszul algebras and quantum groups, Ann. Inst. Fourier (Grenoble) 37 (1987), 191-205

[M2] Yu. I. Manin, Multiparameter quantum deformations of the linear supergroup, Comm. Math. Phys. 123 (1989), 163-175

[M3] Yu. I. Manin, Quantum groups and noncommutative geomtry, CRM, Université de Montréal, QC, 1988

[Mo1] A. I. Molev, Yangians and transvector algebras, Discrete Math. 246 (2002), $231-253$

[Mo2] A. I. Molev, Skew representations of twisted Yangians, Selecta Math. (N.S.) 12 (2006), 1-38

[MG] G. Mühlbach and M. Gasca, A Generalization of Sylvester's Identity on Determinants and Some Applications, Linear Algebra Appl. 66 (1985), 221-234

[V] G. X. Viennot, Heaps of pieces. I: Basic definitions and combinatorial lemmas, Combinatoire énumérative (Montreal, Quebec, 1985), 321-350, Lecture Notes in Math. 1234, Springer, Berlin, 1986

[Z] D. Zeilberger, A combinatorial approach to matrix algebra, Discrete Math. 56 (1985), 61-72 